\title{
Effect of wind turbine converter control on wind power plant harmonic response and resonances
} 10

\section{ISSN 1751-8660}

Received on 24th April 2016 Revised on 15th July 2016 Accepted on 27th September 2016 doi: 10.1049/iet-epa.2016.0241 www.ietdl.org

\section{Q1 Luis Sainz ${ }^{1 凶}$, Lluis Monjo ${ }^{2}$, Joaquin Pedra ${ }^{1}$, Marc Cheah-Mane ${ }^{3}$, Jun Liang ${ }^{3}$, Oriol Gomis-Bellmunt ${ }^{1}$ ${ }^{1}$ Department of Electrical Engineering, ETSEIB-UPC, Av. Diagonal 647, Barcelona 08028, Spain ${ }^{2}$ Department of Industrial and Design System Engineering, ESTCE, Univ. Jaume I, Av. de Vicent Sos Baynat, s/n, 12071 Castelló de la 15 Plana, Spain \\ ${ }^{3}$ School of Engineering, Cardiff University, CF24 3AA, Cardiff, UK $\bowtie$ E-mail: sainz@ee.upc.edu}

Abstract: Wind turbine harmonic emissions due to power electronics are a well-known power quality concern in wind power plants (WPPs). This problem may be increased by resonances because they amplify harmonic distortion around resonance frequencies. Hence, the resonance phenomenon is widely analysed in the literature. Resonance studies usually consider wind turbines (WTs) as ideal current sources, which can lead to inaccurate results. The study explores the effect of WT converter control on WPP harmonic response. It contributes expressions of WT equivalent harmonic impedance including current control of the grid side converter. This impedance allows WT converter control to be considered in WPP modelling to analyse its effect on harmonic response and resonances. PSCAD and Matlab/Simulink simulations were performed to validate the analytical expressions and illustrate the usefulness of the work in predicting harmonic distortions at WT terminals in actual WPPs.

\section{Introduction}

Wind power plants (WPPs) with a large number of wind turbines (WTs) are rapidly developing worldwide [1]. Power quality problems arise in large WPPs because of the harmonic emissions of WTs equipped with power electronics [1-9]. These harmonic emissions are amplified by parallel resonances in the collector grid if they are close to the resonance frequency [1, 6-12]. A summary of the most important WPP harmonic and resonance issues is presented in $[6,8,11]$. In the literature, WPP parallel resonances 40 are analysed to address harmonic concerns [6-12]. Recent works

Q2 also explore resonance influence on stability of WT VSC control $[9,13-15]$. These studies are mainly based on frequency scan analysis, which establishes the frequency range and peak impedance values of resonances. The WT model as an ideal 45 current source is traditionally used in the literature to characterise resonances $[1,6,7]$. However, this model may lead to inaccurate results due to the influence of WT VSC control [6, 8-10]. An impedance-based representation can be used to identify electrical resonances and analyse the influence of grid and VSC control parameters [9, 15-18]. That is why, to obtain more accurate 50 results, WT frequency-dependent models such as Norton equivalent sources, $[9,13-22]$, should be used in WPP resonance studies. In [16-22], the detailed expression of the Norton equivalent impedance of grid-connected VSCs is analytically characterised for resonance and stability studies considering typical one- [19-21] or two- [16-18, 22] cascade control loops in 5 synchronous (or $d-q$ ) reference frame. The numerical results in $[17,18]$ are validated experimentally by the measurement technique in [23]. Most resonance and stability studies consider only the influence of the converter at the point of connection due to the complexity of its Norton equivalent impedance expressions.

60 However, the other VSCs connected to the grid might affect resonance and stability, and therefore they should also be considered. Accordingly, Wang et al. [21] study the harmonic stability of an islanded three-converter system and Bayo-Salas et al. [22] analyses the interaction of two converters connected at different points of the grid. As mentioned in $[19,20]$, resonance characterisation in systems with a large number of converters, such as WPPs, is complex and requires modelling of converters with simplified and aggregated models.

This paper contributes with simplified analytical expressions of WT Norton equivalent impedances for harmonic studies. These expressions, which are validated from PSCAD and Matlab/ Simulink simulations, reduce the complexity of considering WTs when frequency scanning and resonance characterisation is performed in grids with a large number of VSCs such as WPPs. Moreover, this paper explores in detail the effect of WT VSC control on WPP harmonic response and analyses its impact on WPP parallel resonances observed from WTs. The prediction of harmonic distortions at WT terminals in actual WPPs considering the expressions of WT Norton equivalent impedances is also discussed from simulations to show the usefulness of this work in characterising harmonic penetration in actual WPPs.

\section{WPP modelling}

Fig. $1 a$ illustrates a generic offshore WPP layout where type-4 WTs are supplied through medium- to low-voltage (MV/LV) transformers and interconnected with an $N_{\mathrm{r}} \times N_{\mathrm{c}}$ collector grid of medium-voltage (MV) submarine cables from the MV collector bus [6-13]. Type-4 WTs are always equipped with two converters in back-to-back configuration and high-frequency filters are usually installed on the grid side of WT converters to mitigate frequency switching harmonics $[6,7]$. The MV collector bus is connected to the main grid with two high- to medium-voltage (HV/MV) transformers in parallel and a high-voltage (HV) submarine cable. Parallel resonances increase WPP voltage distortions if their frequency is close to the harmonics of WT emissions. Several works study the resonance problem at WT terminals to perform harmonic penetration analysis $[1,6,7]$. To identify parallel resonance frequencies, in harmonic studies WPPs are characterised by their equivalent circuit, Fig. 1b, and the equivalent harmonic impedance observed from the WTs (e.g. $Z_{\mathrm{Eq}, k}$, observed from $\mathrm{WT}_{N r 1}$ in Fig. $1 b)$ is studied. The equivalent harmonic impedances of the main grid, $\underline{Z}_{S, k}, \mathrm{HV} / \mathrm{MV}$ and $\mathrm{MV} / \mathrm{LV}$ transformers, $\underline{Z}_{T, k}, \mathrm{MV}$ underground cables, $\underline{Z}_{L, k}$, and high-frequency filters, $\underline{Z}_{C \mathrm{f}, k}$, must be 


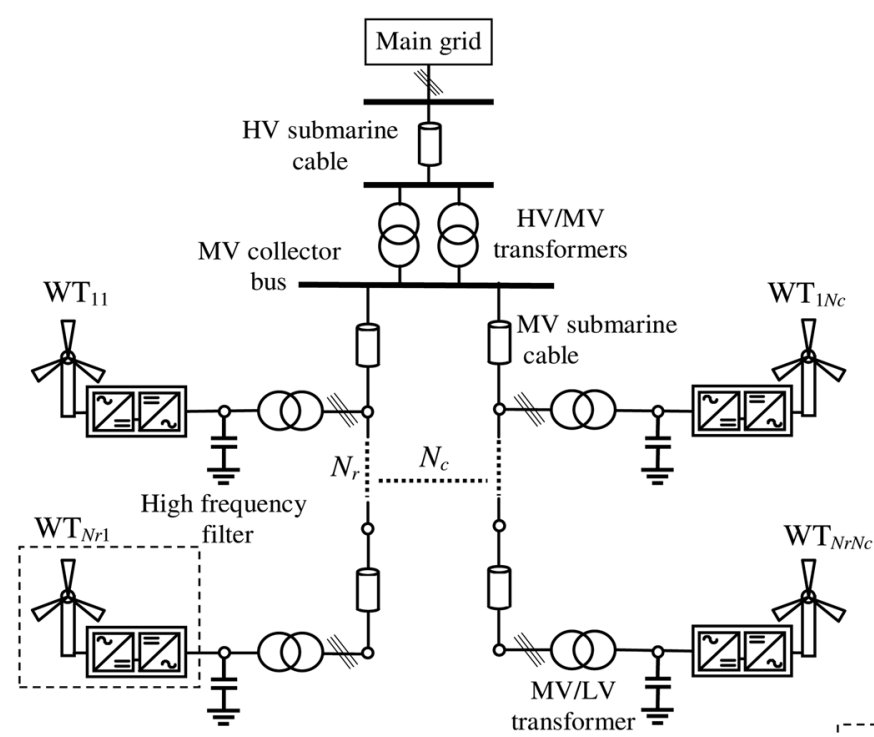

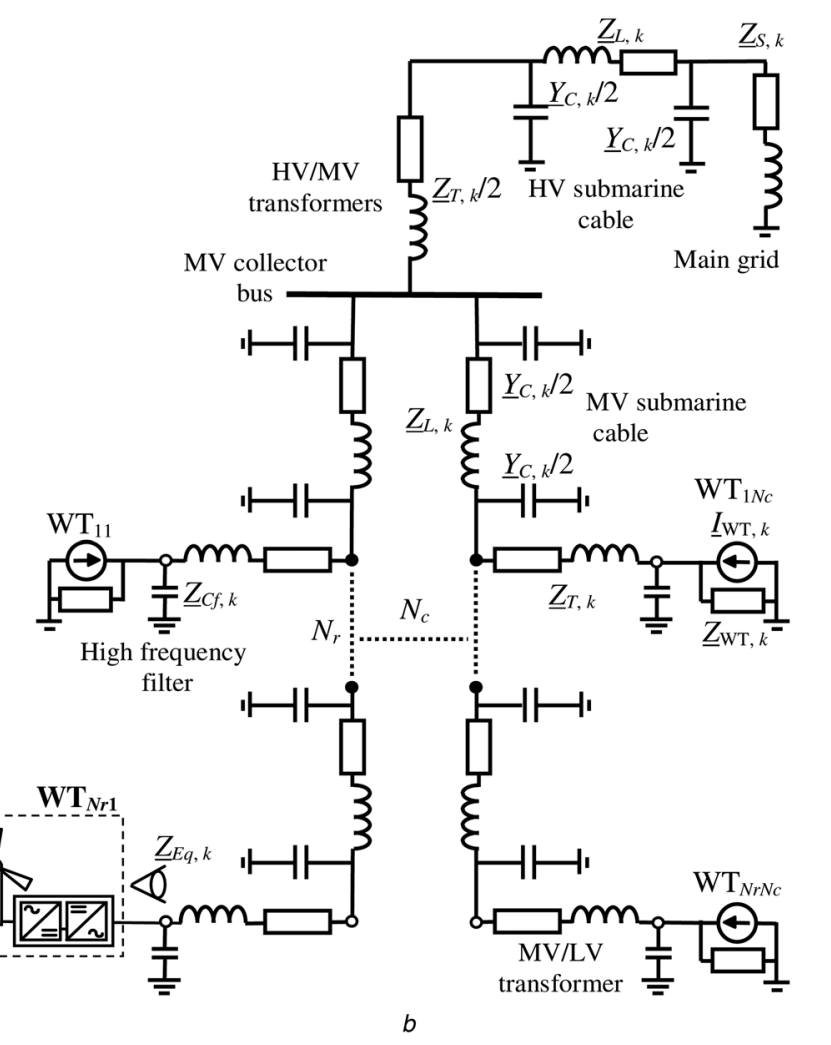

Fig. 1 WPP

$a$ WPP layout

$b$ Equivalent circuit

modelled. These impedances are obtained as follows $[6,7,10]$

$$
\underline{Z}_{S, k}=\frac{U_{O}^{2}}{S_{S}} \frac{1}{\sqrt{1+\tan ^{2} \varphi_{S}}}\left(1+\mathrm{j} k \tan \varphi_{S}\right)
$$$$
\underline{Z}_{T, k}=\varepsilon_{c c} \frac{U_{N, s}^{2}}{S_{N}} \frac{1}{\sqrt{1+\tan ^{2} \varphi_{c c}}}\left(1+\mathrm{j} k \tan \varphi_{c c}\right)
$$$$
\underline{Z}_{L, k}=\underline{Z}_{L x, k} \frac{\sinh \left(\underline{\gamma}_{x, k} D\right)}{\underline{\gamma}_{x, k}} \quad \underline{Y}_{C, k}=\underline{Y}_{C x, k} \frac{\tanh \left(D \underline{\gamma}_{x, k} / 2\right)}{\underline{\gamma}_{x, k} / 2}
$$

$$
\underline{Z}_{C f, k}=-\mathrm{j} \frac{1}{C_{\mathrm{f}} k \omega_{1}},
$$

where $k=f_{k} / f_{1}$ (with $f_{k}$ and $f_{1}$ being the analysed harmonic frequency and the main grid fundamental frequency, respectively), $\omega_{1}=2 \pi \cdot f_{1}$ and, according to Fig. $1 a$,

- $U_{\mathrm{O}}, S_{\mathrm{S}}$ and $\tan \varphi_{\mathrm{S}}$ are the main grid open-circuit voltage, short-circuit power and $X_{\mathrm{S}} / R_{\mathrm{S}}$ ratio at the point of coupling.

- $U_{\mathrm{N}, \mathrm{p}} / U_{\mathrm{N}, \mathrm{s}}, S_{\mathrm{N}}, \varepsilon_{\mathrm{cc}}$ and $\tan \varphi_{\mathrm{cc}}$ are the HV/MV and MV/LV transformer rated primary/secondary voltages and power, per-unit short-circuit impedance and $X_{\mathrm{T}} / R_{\mathrm{T}}$ ratio.

- $\gamma_{x, k}=\left(Z_{L x, k} \cdot \underline{Y}_{C x, k}\right)^{1 / 2}$ is the propagation constant of the cable, $Z_{L x, k}=R_{x}+\mathrm{j} L_{x} k \omega_{1}$ and $Y_{L x, k}=\mathrm{j} C_{x} k \omega_{1}$ are the HV and MV cable distributed parameters and $D$ is the cable length.
Table 1 shows usual offshore WPP parameter values. The WT harmonic model as an ideal current source $\left(\underline{Z}_{\mathrm{WT}, k}=\infty\right.$ in Fig. $\left.1 b\right)$ is typically chosen to perform resonance analysis in harmonic penetration studies because it is only necessary to replace the current sources by an open-circuit at WT terminals [1, 7, 8-12]. However, some works point out that this model might not be accurate due to the possible influence of VSC control on resonances $[6,8,10,13]$. Therefore, WT harmonic models such as Norton equivalent sources are currently proposed for WPP harmonic studies (in particular, resonance studies).

\section{Harmonic model of WTs}

WT VSC control is investigated to determine the equivalent harmonic impedance $Z_{\mathrm{WT}, k}$ of Norton sources, which model WT harmonic behaviour. This frequency-dependent model should be used in frequency scan studies to consider the influence of WT VSC control on resonance frequencies.

\subsection{Expressions of WT equivalent harmonic impedance in balanced conditions}

The WT grid side converter with a filter $Z_{\mathrm{f}}(s)=R_{\mathrm{f}}+L_{\mathrm{f}} \mathrm{s}$ and current control in synchronous ( or $d-q$ ) reference frame are shown in Fig. 2. It must be noted that the converter model in this section only represents the inner current control loop because the outer loops (e.g. the phase-locked loop, PLL, and the direct-voltage controller, DVC) do not affect WPP harmonic response in the $0.5-1.5 \mathrm{kHz}$ frequency range due to their low bandwidths [9]. This assumption allows a VSC symmetrical model that can be characterised with complex impedances or admittances to be obtained. However, the consideration of outer control loops complicates this model unnecessarily, and therefore the WPP harmonic response study. It can be observed that the transfer matrices of the VSC models in $[16,22]$ become the common diagonal matrices of the VSC inner current control loop for frequencies greater than the low bandwidths of the outer control loops. The space phasors are denoted by boldface letters in this study and their components by subscripts $d$ and $q$ (e.g. $\boldsymbol{x}=x_{d}+\mathrm{j} x_{q}$ ). The voltage balance across the filter using $d-q$ complex space phasors in continuous-time $s$ 
Table 1 Offshore WPP parameters

270

275

domain is

$$
\left(R_{\mathrm{f}}+L_{\mathrm{f}} s+\mathrm{j} L_{\mathrm{f}} \omega_{1}\right) \boldsymbol{i}+\boldsymbol{v}=\boldsymbol{v}_{o},
$$

where $v$ is the grid voltage of fundamental angular frequency $\omega_{1}=2 \pi \cdot f_{1}, \boldsymbol{i}$ is the line current and $\boldsymbol{v}_{o}$ is the VSC terminal voltage. The converter voltage reference can be determined as

$$
\boldsymbol{v}_{\text {ref }}=F_{\text {PI }}(s)\left(\boldsymbol{i}_{\text {ref }}-H_{i}(s) \boldsymbol{i}\right)+\mathrm{j} L_{\mathrm{f}} \omega_{1} H_{i}(s) \boldsymbol{i}+H_{v}(s) \boldsymbol{v} .
$$

The voltage $\boldsymbol{v}_{o}$ generated by the VSC and the converter voltage reference $v_{\text {ref }}$ are commonly related to the transfer function of converter time delay $D(s)$ (i.e. $\boldsymbol{v}_{o}=D(s) \cdot v_{\mathrm{ref}}$ ). A negligible converter time delay (i.e. $D(s)=1$ and therefore $\boldsymbol{v}_{o}=\boldsymbol{v}_{\text {ref }}$ ) is considered in the below characterisation of the WT equivalent harmonic impedances, but the influence of this delay on the impedances is discussed in Section 3.4.2. In (3), $\boldsymbol{i}_{\mathrm{ref}}$ is the converter reference current, and a feedback of the line current through a PI controller with transfer function $F_{\mathrm{PI}}(s)$ is used

$$
F_{\mathrm{PI}}(s)=K_{\mathrm{p}}+\frac{K_{\mathrm{i}}}{s},
$$

where $K_{\mathrm{p}}$ and $K_{\mathrm{i}}$ are the PI control proportional and integral gains, respectively. Moreover, the influence of feedback and feedforward signal filtering on measured line current and grid voltage signals is also considered through low-pass filter transfer functions $H_{i}(s)$ and $H_{v}(s)[14,16]$

$$
H_{x}(s)=\frac{\boldsymbol{x}_{m}}{\boldsymbol{x}}=\frac{\alpha_{\mathrm{f} x}}{s+\alpha_{\mathrm{f} x}} \quad(\boldsymbol{x}=\boldsymbol{i}, \boldsymbol{v}),
$$

where $\boldsymbol{x}$ represents the line current and grid voltage signals, $\boldsymbol{x}_{m}$ represents the output filters and $\alpha_{\mathrm{f} x}$ is the bandwidth of

310 the low-pass filters. For high bandwidths, $\boldsymbol{x}$ is unfiltered (i.e. $H_{x}(s) \simeq 1$, and therefore $\left.\boldsymbol{x}_{m} \simeq \boldsymbol{x}\right)$. For low bandwidths, $\boldsymbol{x}$ is

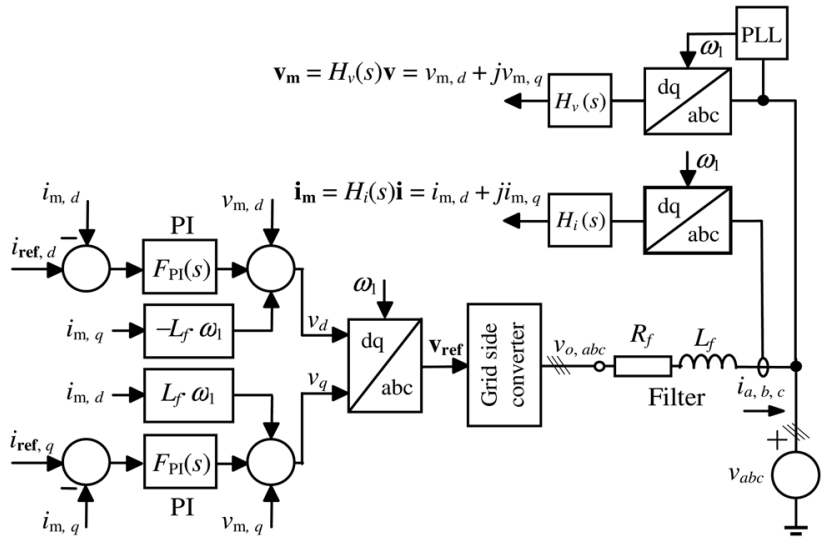

Fig. 2 General structure for VSC current control in synchronous (or $d-q$ ) 330 reference frame completely filtered (i.e. $H_{x}(s) \simeq 0$, and therefore $\boldsymbol{x}_{m} \simeq \mathbf{0}$ ). In [16], the control design results in $K_{\mathrm{p}}=\alpha_{\mathrm{c}} L_{\mathrm{f}}$ and $K_{\mathrm{i}}=\alpha_{\mathrm{c}} R_{\mathrm{f}}$, where $\alpha_{\mathrm{c}}$ is the current controller bandwidth. This bandwidth should verify $\alpha_{\mathrm{c}} \leq 0.2 \cdot\left(2 \pi f_{\mathrm{s}}\right)$, with $f_{\mathrm{s}}$ being the converter switching frequency, which is usually $>1 \mathrm{kHz}$ for modern VSCs [16]. The recommended bandwidth of the grid voltage low-pass filter is $\alpha_{\mathrm{f} v} \leq 0.1 \alpha_{\mathrm{c}}$ for normal-mode operation and $\alpha_{\mathrm{f} v} \geq \alpha_{\mathrm{c}}$ for transient-mode operation. The bandwidth of the line current feedback low-pass filter is supposed to be large enough compared with the current controller bandwidth to ensure that the filter does not affect the controller response. A line current low-pass filter bandwidth five times higher than the current controller bandwidth is considered in the study (i.e. $\alpha_{\mathrm{f} i} \geq 5 \alpha_{\mathrm{c}}$ ).

The WT equivalent harmonic impedance can be obtained by considering the $d-q$ complex space phasor expressions of three-phase non-sinusoidal quantities in balanced conditions (see Appendix) and combining the voltage balance across the filter (2) and the converter voltage reference (3) in frequency domain. By relating (2) and (3) by means of converter time delay, the following expression is obtained

$$
\begin{aligned}
& \left(R_{\mathrm{f}}+L_{\mathrm{f}} s+\mathrm{j} L_{\mathrm{f}} \omega_{1}\right) \boldsymbol{i}+\boldsymbol{v}=D(s) \\
& \left(F_{\mathrm{PI}}(s)\left(\boldsymbol{i}_{\mathrm{ref}}-H_{i}(s) \boldsymbol{i}\right)+\mathrm{j} L_{\mathrm{f}} \omega_{1} H_{i}(s) \boldsymbol{i}+H_{v}(s) \boldsymbol{v}\right),
\end{aligned}
$$

which can be arranged as

$$
\begin{aligned}
& \boldsymbol{v}\left(1-D(s) H_{v}(s)\right)=D(s) F_{\mathrm{PI}}(s) \boldsymbol{i}_{\text {ref }} \\
& \quad-\left(R_{\mathrm{f}}+L_{\mathrm{f}} s+\mathrm{j} L_{\mathrm{f}} \omega_{1}+D(s) H_{i}(s)\left(F_{\mathrm{PI}}(s)-\mathrm{j} L_{\mathrm{f}} \omega_{1}\right)\right) \boldsymbol{i} .
\end{aligned}
$$

Considering that the positive $(k=4,7 \ldots)$ and negative $(k=2,5 \ldots)$ sequence harmonic phasors of balanced three-phase non-sinusoidal voltages and currents, $\underline{X}_{k}$ (with $X=V$ or $I$ ), are expressed in $d-q$ frame as the following complex space phasors (Appendix):

- Positive sequence components: $\boldsymbol{x}=x_{d}+\mathrm{j} x_{q}=\underline{X}_{k} \cdot \mathrm{e}^{\mathrm{j}(k-1) \omega_{1} t}$ for $k=4,7$..

- Negative sequence components: $\boldsymbol{x}=x_{d}-\mathrm{j} x_{q}=\underline{X}_{k}^{*} \cdot \mathrm{e}^{-\mathrm{j}(k+1) \omega_{1} t}$ for $k=2,5$..

The transformations $s \rightarrow(k-1) \omega_{1}$ for the positive-sequence components $\left(X_{k}\right.$ with $\left.k=4,7 \ldots\right)$ and $s \rightarrow-(k+1) \omega_{1}$ for the negative-sequence components $\left(X_{k}\right.$ with $\left.k=2,5 \ldots\right)$ are applied to express (7) in harmonic steady state, i.e.

$$
\begin{aligned}
& \underline{V}_{k}\left(1-\underline{D}_{h} \underline{H}_{v, h}\right)=\underline{D}_{h} \underline{F}_{\mathrm{P}, h} \underline{I}_{\mathrm{ref} k} \\
& \quad+\left(R_{\mathrm{f}}+j L_{\mathrm{f}} h \omega_{1}+j L_{\mathrm{f}} \omega_{1}+\underline{D}_{h} \underline{H}_{i, h}\left(\underline{F}_{\mathrm{P} \mathrm{I}, h}-j L_{\mathrm{f}} \omega_{1}\right)\right) \\
& \quad\left(-\underline{I}_{k}\right) \quad(h=k-1) \\
& \underline{V}_{k}^{*}\left(1-\underline{D}_{h} \underline{H}_{v, h}\right)=\underline{D}_{h} \underline{\mathrm{F}}_{\mathrm{P} \mathrm{I}, \underline{I}_{\mathrm{ref} k}} \\
& \quad+\left(R_{\mathrm{f}}+j L_{\mathrm{f}} h \omega_{1}+j L_{\mathrm{f}} \omega_{1}+\underline{D}_{h} \underline{H}_{i, h}\left(\underline{F}_{\mathrm{P} \mathrm{I}, h}-j L_{\mathrm{f}} \omega_{1}\right)\right) \\
& \quad\left(-\underline{I}_{k}^{*}\right) \quad(h=-(k+1)),
\end{aligned}
$$

where 
- $\underline{V}_{k}, \underline{I}_{k}$ and $\underline{I}_{\text {refk }}$ are the $k$ th harmonic phasors of the grid voltage $\boldsymbol{v}$, line current $\boldsymbol{i}$ and converter reference current $\boldsymbol{i}_{\text {ref }}$ (superscript $*$ indicates complex conjugate).

- $\underline{F}_{\mathrm{PI}, h}, \underline{H}_{i, h}$ and $\underline{H}_{v, h}$ represent the frequency response of the PI controller and feedback and feedforward low-pass filters, which are obtained by setting $s \rightarrow \mathrm{j} h \omega_{1}$ in their corresponding transfer functions (4) and (5), i.e.

$$
\begin{gathered}
\underline{F}_{\mathrm{PI}, h}=\underline{F}_{\mathrm{PI}}\left(\mathrm{j} h \omega_{1}\right)=K_{\mathrm{p}}+\frac{K_{\mathrm{i}}}{\mathrm{j} h \omega_{1}}=K_{\mathrm{p}}-\mathrm{j} \frac{K_{\mathrm{i}}}{h \omega_{1}} \\
\underline{H}_{x, h}=\underline{H}_{x}\left(\mathrm{j} h \omega_{1}\right)=\frac{\alpha_{\mathrm{f} x}}{\mathrm{j} h \omega_{1}+\alpha_{\mathrm{f} x}}=\frac{\alpha_{\mathrm{f} x, r}}{\mathrm{j} h+\alpha_{\mathrm{f} x, r}}, \\
\alpha_{\mathrm{f} x, r}=\frac{\alpha_{\mathrm{f} x}}{\omega_{1}} \quad(x=i, v) .
\end{gathered}
$$

Note that $H_{x, h}(s) \simeq 1$ for high bandwidths, with $\alpha_{\mathrm{f} x, r}>>h$, and

$H_{x, h}(s) \simeq 0$ for low bandwidths, with $\alpha_{\mathrm{fx}, r}<<h$ (e.g. $\left.\alpha_{\mathrm{f} x, r} \simeq 1\right)$.

- $D_{h}$ represents the frequency response of converter time delay, which is considered negligible in the below study, i.e. $\underline{D}_{h}=1$.

Considering (8) and assuming that the harmonic phasors of the converter reference current are equal to zero (i.e. $\underline{I}_{\text {refk }}=0$ ), the WT equivalent harmonic impedance is obtained from the ratio of the grid voltage and line current phasors, i.e. $\underline{Z}_{\mathrm{WT}, k}=R_{\mathrm{WT}, k}+\mathrm{j} X_{\mathrm{WT}, k}=$ $V_{k} /\left(-I_{k}\right)$, where the negative sign is due to the current direction definition outside the converter. Thus, the WT equivalent harmonic impedance of the positive-sequence harmonic components becomes

$$
\begin{gathered}
\underline{Z}_{\mathrm{WT}, p k}=\frac{R_{\mathrm{f}}+\mathrm{j} L_{\mathrm{f}}(h+1) \omega_{1}+\underline{H}_{i, h}\left(\underline{F}_{\mathrm{PI}, h}-\mathrm{j} L_{\mathrm{f}} \omega_{1}\right)}{1-\underline{H}_{v, h}} \\
(h=k-1 ; \quad k=4,7 \ldots),
\end{gathered}
$$

and, according to (36), the WT equivalent harmonic impedance for the negative-sequence harmonic components in (8) corresponds to the conjugate expression of the WT equivalent harmonic impedance for the positive-sequence harmonic components, with $h=-(k+1)$. The above WT equivalent impedances are obtained for the typical one-cascade control loop in Fig. 2. Other converter control structures lead to different WT Norton equivalent impedances which could be characterised by the same procedure. In the following subsections, the WT equivalent harmonic impedance in (10) is determined in three successive steps from the simplest to the most complex case to enhance the clarity of the study and analyse the impact of WT VSC control on the equivalent impedance.

3.1.1 Unfiltered line current and grid voltage (case A): This study is restricted to the simplest control, where the line current and grid voltage harmonics are unfiltered (i.e. $H_{x, h} \simeq 1$ for $x=i, v$ ). In this case, the positive-sequence harmonic component expression in (8) can be rewritten as follows

$$
\begin{gathered}
0=\left(R_{\mathrm{f}}+\mathrm{j} L_{\mathrm{f}} \omega_{1} h+\underline{F}_{\mathrm{PI}, h}\right)\left(-\underline{I}_{k}\right) \\
(h=k-1 ; k=4,7 \ldots),
\end{gathered}
$$

which implies that the $k$ th harmonic phasors $\underline{I}_{k}$ of the WT line currents for the positive-sequence are equal to zero, and therefore the WT equivalent harmonic impedance becomes $\underline{Z}_{\mathrm{WT}, k}^{(A)}=\infty(k=$ $4,7 \ldots)$. This is also true for the negative-sequence harmonic components $(k=2,5 \ldots)$. In this way, the $d-q$ reference frame control with unaffected feedback and feedforward grid signals can be modelled for the positive- and negative-sequence harmonic components as the typical ideal harmonic current source used in the literature.

3.1.2 Unfiltered line current (case B): In this case, the line current harmonics are unfiltered and the grid voltage harmonics are filtered (i.e. $H_{i, h} \simeq 1$ and $0 \leq H_{v, h}<1$ ). The positive-sequence harmonic component expression in (8) can be rewritten as follows:

$$
\begin{aligned}
& \underline{V}_{k}\left(1-\underline{H}_{v, h}\right)=\left(R_{\mathrm{f}}+\mathrm{j} L_{\mathrm{f}} \omega_{1} h+\underline{F}_{\mathrm{PI}, h}\right)\left(-\underline{I}_{k}\right) \\
& \quad(h=k-1 ; \quad k=4,7 \ldots),
\end{aligned}
$$

and the WT equivalent harmonic impedance of the 475 positive-sequence harmonic components becomes

$$
\begin{aligned}
\underline{Z}_{\mathrm{WT}, p k}^{(B)}= & \frac{R_{\mathrm{f}}+\mathrm{j} L_{\mathrm{f}} h \omega_{1}+\underline{F}_{\mathrm{PI}, h}}{1-\underline{H}_{v, h}}=R_{\mathrm{f}}+K_{\mathrm{p}}+\alpha_{\mathrm{f} v, r}\left(L_{\mathrm{f}} \omega_{1}-\frac{K_{\mathrm{i}}}{h^{2} \omega_{1}}\right) \\
& +\mathrm{j}\left(L_{\mathrm{f}} \omega_{1} h-\frac{K_{\mathrm{i}}}{h \omega_{1}}-\frac{\alpha_{\mathrm{f} v, r}}{h}\left(R_{\mathrm{f}}+K_{\mathrm{p}}\right)\right) \\
& (h=k-1) .
\end{aligned}
$$

The WT equivalent harmonic impedance of the negative-sequence harmonic components $(k=2,5 \ldots)$ corresponds to the conjugate expression of the positive-sequence WT equivalent harmonic impedance (13), with $h=-(k+1)$.

If the grid voltage harmonics are completely filtered (i.e. $\alpha_{\mathrm{fv}, r}$ is small enough for $H_{v, h} \simeq 0$ ), the WT equivalent harmonic impedance for the positive- and negative-sequences in (13) becomes

$$
\begin{aligned}
& \underline{Z}_{\mathrm{WT}, k}^{(B)}=R_{\mathrm{f}}+K_{\mathrm{p}}+\mathrm{j}\left(L_{\mathrm{f}} h \omega_{1}-\frac{K_{\mathrm{i}}}{h \cdot \omega_{1}}\right) \\
& (h=k-1 ; k=4,7 \ldots) \\
& (h=k+1 ; k=2,5 \ldots)
\end{aligned}
$$

3.1.3 Filtered line current and grid voltage (case C): In this case, the line current and the grid voltage harmonics are filtered. The currents are filtered with a bandwidth five times greater than the current controller bandwidth (i.e. $\alpha_{\mathrm{f} i} \geq 5 \alpha_{\mathrm{c}}$ and therefore $\alpha_{\mathrm{fi}, r} \geq 15$ ), while the voltages can be completely filtered (i.e. $0 \leq H_{v, h}<1$ ). The positive-sequence harmonic component expression in (8) can be rewritten as

$$
\begin{aligned}
& \underline{V}_{k}\left(1-\underline{H}_{v, h}\right)=\left(R_{\mathrm{f}}+j L_{\mathrm{f}}(h+1) \omega_{1}+\underline{F}_{\mathrm{PI}, h} \underline{H}_{i, h}-\mathrm{j} L_{\mathrm{f}} \omega_{1} \underline{H}_{i, h}\right) \\
& \left(-\underline{I}_{k}\right) \quad(h=k-1 ; k=4,7 \ldots),
\end{aligned}
$$

and the WT equivalent harmonic impedance of the positive-sequence harmonic components in (10) is obtained, which can be arranged as

$$
\underline{Z}_{\mathrm{WT}, p k}^{(C)}=\frac{h A_{k}+\alpha_{\mathrm{f} v, r} B_{k}+j\left(h B_{k}-\alpha_{\mathrm{f} v, r} A_{k}\right)}{h\left(\alpha_{\mathrm{f}, r}^{2}+h^{2}\right)} \quad(h=k-1),
$$

where

$$
\begin{aligned}
& A_{k}=R_{\mathrm{f}}\left(\alpha_{\mathrm{f} i, r}^{2}+h^{2}\right)+\alpha_{\mathrm{f} i, r}^{2} K_{\mathrm{p}}-\alpha_{\mathrm{f} i, r}\left(L_{\mathrm{f}} h \omega_{1}+\frac{K_{\mathrm{i}}}{\omega_{1}}\right) \\
& B_{k}=h\left(L_{\mathrm{f}} h(h+1) \omega_{1}-\alpha_{\mathrm{f} i, r} K_{\mathrm{p}}\right)+\alpha_{\mathrm{f} i, r}^{2}\left(L_{\mathrm{f}} h \omega_{1}-\frac{K_{\mathrm{i}}}{h \omega_{1}}\right) .
\end{aligned}
$$

The WT equivalent harmonic impedance of the negative-sequence harmonic components $(k=2,5 \ldots)$ corresponds to the conjugate expression of the positive-sequence WT equivalent harmonic 

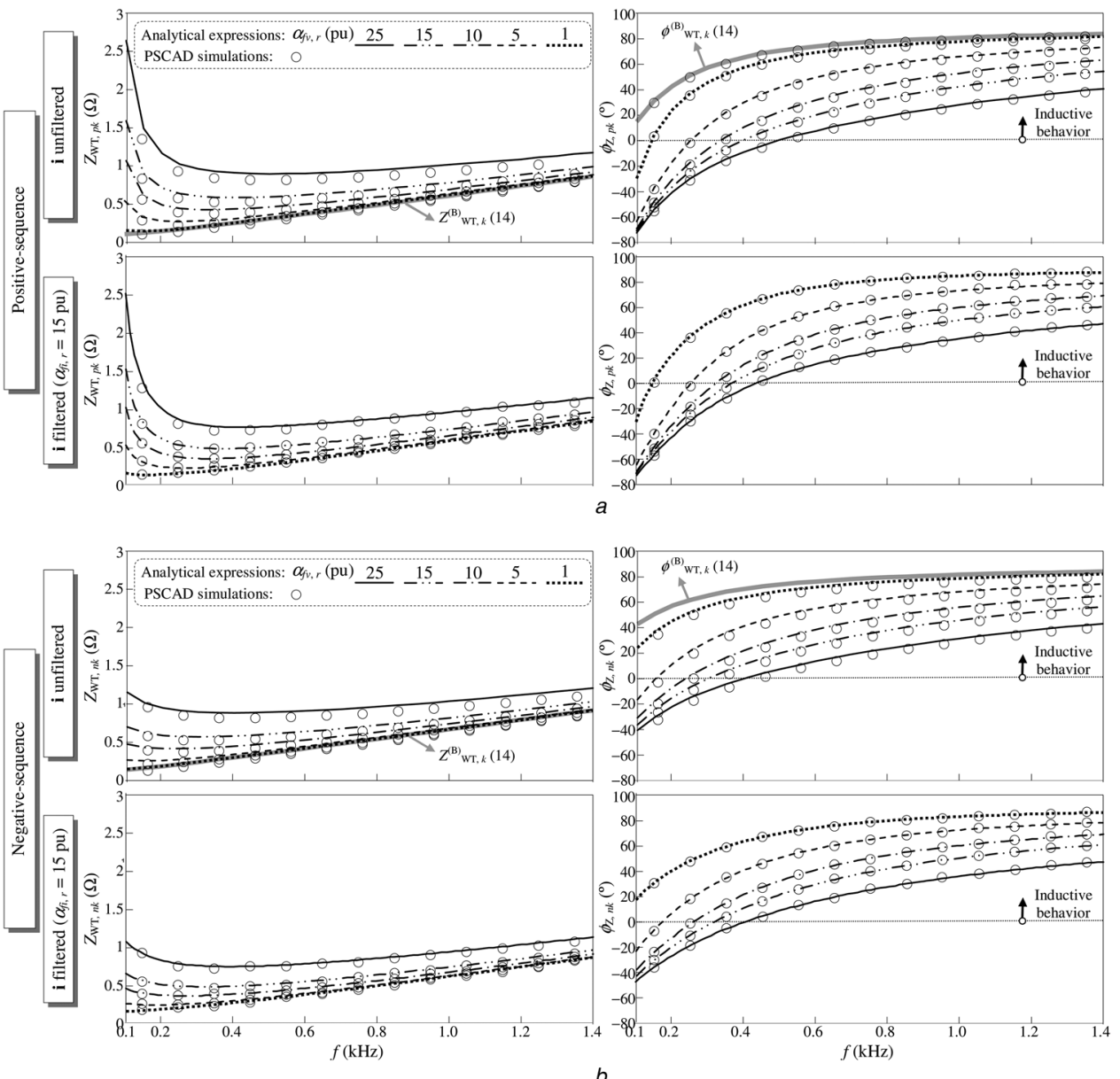

Fig. 3 Magnitude (left) and phase angle (right) of the WT equivalent harmonic impedance (data in Table 1 and D(s) =1)

$a$ Positive sequence

$b$ Negative sequence

impedance (16), with $h=-(k+1)$. The WT equivalent harmonic impedances in Sections 3.1.1 and 3.1.2 can be derived from (16).

\subsection{Validation of the WT equivalent harmonic impedance}

Considering the data in Table 1, Fig. 3 shows the magnitude and phase angle results of the WT equivalent harmonic impedance obtained from the analytical expressions in (16), i.e.

$$
\underline{Z}_{\mathrm{WT}, k}^{(C)}=Z_{\mathrm{WT}, k} e^{\mathrm{j} \phi_{\mathrm{WT}, k}}=\frac{\sqrt{\left(\alpha_{\mathrm{f} v, r}^{2}+h^{2}\right)\left(A_{k}^{2}+B_{k}^{2}\right)}}{h\left(\alpha_{\mathrm{f}, r}^{2}+h^{2}\right)}
$$$$
e^{\mathrm{jtan}^{-1}\left(\left(h B_{k}-\alpha_{\mathrm{fv}, r} A_{k}\right) /\left(h A_{k}+\alpha_{\mathrm{fv}, r} B_{k}\right)\right)} \text {. }
$$

The results in Fig. 3 are in agreement with [9, 15, 17, 18, 23]. To the accuracy of the alytical study, a PSCAD frequency scan is numerically performed on the WT grid side converter in Fig. 2. This frequency scan is obtained by applying the following steps:

- A small voltage disturbance (e.g. $0.1 \mathrm{pu}$ ) at a certain frequency is applied to the supply voltage of the WT grid side converter.

- The steady-state voltage and current values of one phase are measured and the harmonic components of the disturbance frequency are obtained.
- The impedance of the circuit at the disturbance frequency is calculated from the above harmonic voltage and current values.

The results are shown with dots in Fig. 3, where only the dominant harmonics in WPPs (i.e. $k=7,13 \ldots$ for the positive-sequence and $k=$ $5,11 \ldots$ for the negative-sequence) are plotted for the sake of clarity. The above results could be comparable with the equivalent impedance characterised in $[15,17,18]$ for stability studies. However, the present study only focuses on the harmonic frequencies of WT emissions, which allows simpler expressions for discussing the impact of WT VSC control on WPP resonances to be obtained. Fig. 3 shows that the WT equivalent harmonic impedance (in particular, its resistive component) is mainly affected by the grid voltage low-pass filter bandwidth while the impact of the line current low-pass filter bandwidth is small. The details of these influences are analysed in the next sections. The WT grid side converter harmonic currents in the PSCAD simulations of the unfiltered control case (case A) are equal to zero, which agrees with $\underline{Z}_{\mathrm{WT}, k}^{(A)}=\infty$.

Experimental validation is out of the scope of the paper because access to actual WTs to perform measurements was not possible. However, WT equivalent harmonic impedance could be measured by one of the three impedance measurement methods proposed in [23]. These methods are similar to the numerical procedure applied in the PSCAD frequency scan. A frequency response analyser is used to supply VSC with a small voltage disturbance at a specific frequency and the positive- and negative-sequence components of the VSC impedance are obtained from measurements of two-phase currents and voltage disturbance. 
Table 2 Simplified expressions of the WT equivalent harmonic impedance $\underline{Z}_{\mathrm{WT}, k}=R_{\mathrm{WT}, \mathrm{k}}+\mathrm{j} X_{\mathrm{WT}, \mathrm{k}}\left(f_{l m}<1 \mathrm{kHz}\right)$ without considering converter time delay $(D$ $(s)=1)$

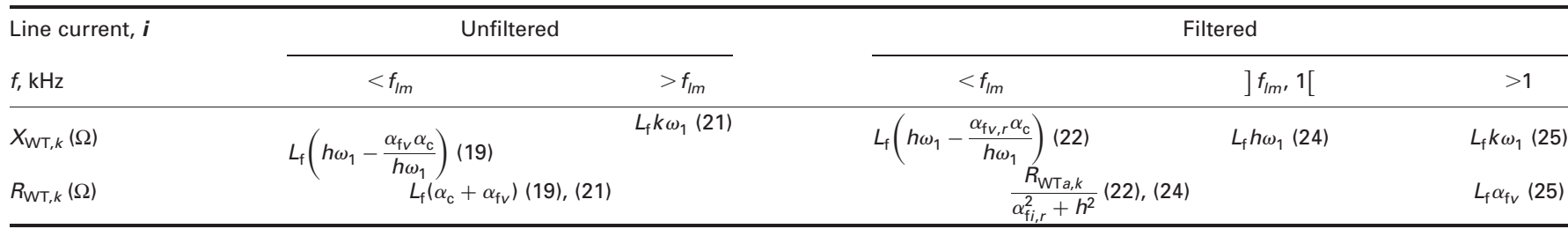

Note: $h=k-1 ; k=4,7 \ldots$ and $h=k+1 ; k=2,5 \ldots, R_{\mathrm{WT} a, k}(23)$

Subsequently, symmetrical transformation is applied to the measurements to obtain the sequence components of the WT equivalent harmonic impedances. According to $[17,18]$, measurements allow analytical and numerical results to be validated.

\subsection{Simplified expressions of the WT equivalent harmonic impedance}

Simplified expressions of the WT positive- and negative-sequence equivalent harmonic impedance are obtained when the currents are unfiltered and filtered. The simplifications consider that the filter resistance $R_{\mathrm{f}}$ and the PI control integral gain $K_{\mathrm{i}}=\alpha_{\mathrm{c}} R_{\mathrm{f}}$ can be neglected because of their small value $[14,15]$. The relation between the filter inductance and the PI control proportional gain, $K_{\mathrm{p}}=\alpha_{\mathrm{c}} L_{\mathrm{f}}$, is also considered to obtain more compact expressions.

3.3.1 Unfiltered line currents: The WT positive- and negative-sequence equivalent harmonic impedance can be approximated from (13) as

$$
\begin{aligned}
\underline{Z}_{\mathrm{WT}, k} & \simeq K_{\mathrm{p}}+L_{\mathrm{f}} \alpha_{\mathrm{f} v, r} \omega_{1}+\mathrm{j}\left(L_{\mathrm{f}} h \omega_{1}-\frac{\alpha_{\mathrm{f} v, r}}{h} K_{\mathrm{p}}\right) \\
& =L_{\mathrm{f}}\left(\alpha_{\mathrm{c}}+\alpha_{\mathrm{f} v}\right)+\mathrm{j} L_{\mathrm{f}}\left(h \omega_{1}-\frac{\alpha_{\mathrm{f} v} \alpha_{\mathrm{c}}}{h \omega_{1}}\right) \\
& (h=k-1 ; k=4,7 \ldots \text { and } h=k+1 ; k=2,5 \ldots) .
\end{aligned}
$$

The WT equivalent harmonic impedance in (19) shows an inductive behaviour (see unfiltered cases in Fig. 3) for frequencies above $f_{l m}$

$$
\operatorname{Im}\left\{\underline{Z}_{\mathrm{WT}, k}\right\}=h \omega_{1}-\frac{\alpha_{\mathrm{fv}} \alpha_{\mathrm{c}}}{h \omega_{1}}>0 \Rightarrow f>f_{l m}=f_{1}+\frac{\sqrt{\alpha_{\mathrm{c}} \alpha_{\mathrm{f} v}}}{2 \pi},
$$

and this impedance can be approximated as follows:

$$
\begin{gathered}
\underline{Z}_{\mathrm{WT}, k} \simeq L_{\mathrm{f}}\left(\alpha_{\mathrm{c}}+\alpha_{\mathrm{f} v}\right)+\mathrm{j} L_{\mathrm{f}} k \omega_{1} \quad(k=4,7 \ldots \quad \text { and } \\
k=2,5 \ldots) .
\end{gathered}
$$

Therefore, the WT equivalent harmonic impedance when the line current is unfiltered is always passive with a positive resistance affected by the current control and grid voltage low-pass filter bandwidths $\left(\alpha_{\mathrm{c}}\right.$ and $\left.\alpha_{\mathrm{fv}}\right)$. Moreover, for frequencies above $f_{l m}$, the imaginary part of this impedance can be simplified as an inductance approximately equal to the filter inductance of the grid-side converter. The frequency $f_{l m}$ is usually $<500 \mathrm{~Hz}$ for typical WT parameters (Table 1).

3.3.2 Filtered line currents: The WT positive- and negative-sequence equivalent harmonic impedance can be approximated from (16) as

$$
\begin{aligned}
\underline{Z}_{\mathrm{WT}, k} & \simeq \frac{R_{\mathrm{WT} a, k}}{\alpha_{\mathrm{f} i, r}^{2}+h^{2}}+\mathrm{j}\left(L_{\mathrm{f}} h \omega_{1}-\frac{\alpha_{\mathrm{f} v, r}}{h} K_{\mathrm{p}}\right) \\
& =\frac{R_{\mathrm{WT} a, k}}{\alpha_{\mathrm{f} i, r}^{2}+h^{2}}+\mathrm{j} L_{\mathrm{f}}\left(h \omega_{1}-\frac{\alpha_{\mathrm{f} v, r} \alpha_{\mathrm{c}}}{h \omega_{1}}\right) \\
& (h=k-1 ; \quad k=4,7 \ldots \quad \text { and } \quad h=k+1 ; \quad k=2,5 \ldots),
\end{aligned}
$$

with (see (23))

For frequencies above $f_{l m}$, the imaginary part of the impedance can be approximated as an inductance, similarly to the unfiltered case, i.e.

$$
\underline{Z}_{\mathrm{WT}, k} \simeq \frac{R_{\mathrm{WT} a, k}}{\alpha_{\mathrm{f}, r}^{2}+h^{2}}+\mathrm{j} L_{\mathrm{f}} k \omega_{1} \quad \begin{aligned}
& (h=k-1 ; \quad k=4,7 \ldots) \\
& (h=k+1 ; \quad k=2,5 \ldots)
\end{aligned},
$$

and, for frequencies above $1 \mathrm{kHz}$ (i.e. $k>20$ ), (24) can be approximated as follows

$$
\begin{aligned}
& \underline{Z}_{\mathrm{WT}, k} \simeq \frac{L_{\mathrm{f}}\left(k \omega_{1} \alpha_{\mathrm{fv}, r} k+\alpha_{\mathrm{f} v, r} \alpha_{\mathrm{f} i, r}^{2} \omega_{1}\right)}{\alpha_{\mathrm{f} i, r}^{2}+k^{2}} \\
& \quad+\mathrm{j} L_{\mathrm{f}} k \omega_{1}=L_{\mathrm{f}} \alpha_{\mathrm{f} v}+\mathrm{j} L_{\mathrm{f}} k \omega_{1} \quad(k=4,7 \ldots \quad \text { and } \quad k=2,5 \ldots),
\end{aligned}
$$

where the resistance $R_{\mathrm{WT} a, k}$ in (21) tends to $L_{\mathrm{f}} \alpha_{\mathrm{fv}}$, which is graphically verified in Section 3.3.3.

3.3.3 Summary of expressions: Considering $f_{l m}<1 \mathrm{kHz}$, Table 2 summarises the expressions proposed for the WT equivalent harmonic impedance. To check these expressions, Fig. 4 shows the positive-sequence WT equivalent resistance and reactance $\left(R_{\mathrm{WT}, k}\right.$ and $X_{\mathrm{WT}, k}$ with $\left.k=4,7 \ldots\right)$ for unfiltered and filtered $\left(\alpha_{\mathrm{f} i, r}=15 \mathrm{pu}\right)$ line current and different $\alpha_{\mathrm{fv}, r}$ values. The resistance is equal to $L_{\mathrm{f}}\left(\alpha_{\mathrm{c}}+\alpha_{\mathrm{fv}}\right)$ in the unfiltered case, whereas it becomes $L_{\mathrm{f}} \alpha_{\mathrm{fv}}$ for frequencies $>1 \mathrm{kHz}$ in the filtered case. It is also observed that the reactance tends to $L_{\mathrm{f}} \omega_{1}$ for frequencies above $f_{l m}$ in both unfiltered and filtered cases.

\subsection{Influence of unbalanced voltage conditions and converter time delay}

The impact of unbalanced voltage conditions and converter time delay on WT equivalent harmonic impedance is analysed in the following subsections.

3.4.1 Unbalanced voltage conditions: Like in balanced conditions, the positive- and negative-sequence WT equivalent harmonic impedance can be obtained by combining (2) and (3) in

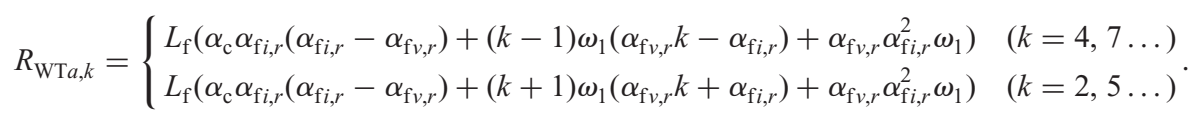




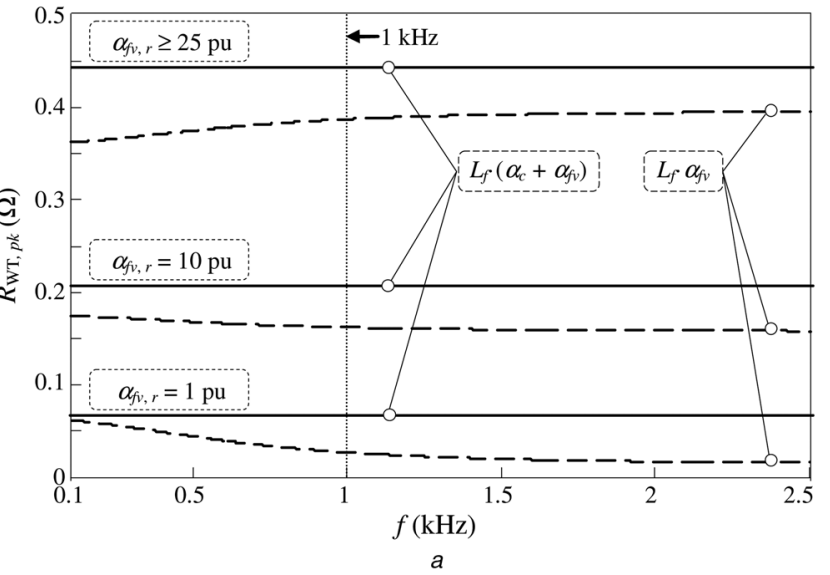

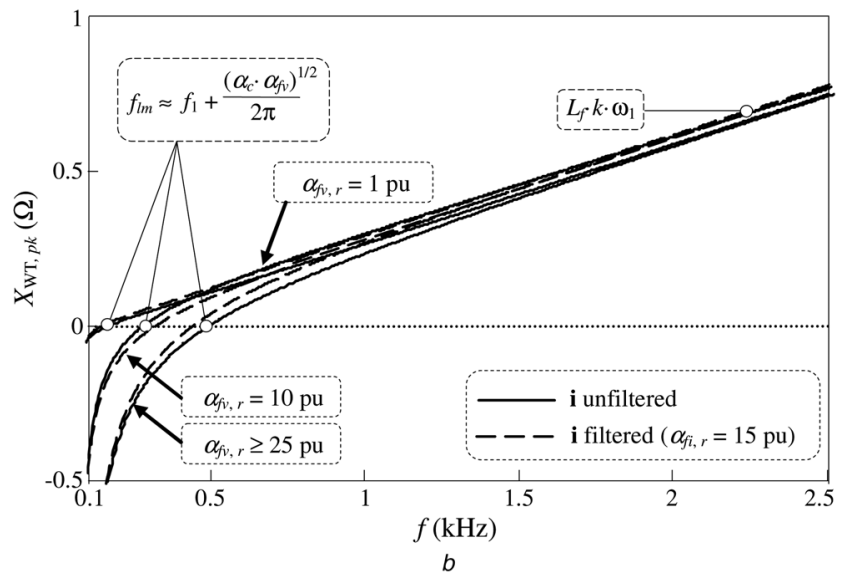

860

865

870

Fig. 4 Study of the WT positive-sequence equivalent impedance (data in Table 1 and $D(s)=1$ )

$810 a$ WT equivalent resistance

$b$ WT equivalent frequency domain and considering that the $d-q$ complex space phasors of three-phase non-sinusoidal voltages in unbalanced

815 conditions contain positive- and negative-sequence components for all harmonics (34). Thus, the expressions of the WT equivalent harmonic impedance in unbalanced conditions are the same as in balanced conditions, except that the positive- and negative-sequence must be considered for all harmonics $(k>1)$ instead of the positive-sequence impedance for $k=4,7 \ldots$ and the negative for $k=2,5 \ldots$

Another effect of unbalanced supply voltages is that the negative-sequence component of the fundamental $d-q$ space phasor has a second harmonic oscillation, i.e. the negative-sequence component is translated to twice the

825 fundamental frequency (34), which can affect grid synchronisation of the PLL. This can lead to coupling equivalent harmonic impedances $Z_{\mathrm{WT}, \mathrm{pn} k}$ between positive- and negative-sequence voltages and currents $[18,23]$. However, these harmonic coupling terms can be neglected for typical PLL designs not exceeding a few tens of hertz [18].

3.4.2 Converter time delay: Converter time delay between the voltage $v_{o}$ generated by the VSC and the converter voltage reference $v_{\text {ref }}$ is typically considered as $D(s)=\mathrm{e}^{-s T_{\mathrm{d}}}$, where $T_{\mathrm{d}}$ is 835 the time delay [14]. This time delay is approximately composed of one sampling period of computation delay and half a sampling period of modulation delay (i.e. switching process) and is given by $T_{\mathrm{d}} \simeq 1.5 T_{\mathrm{s}}$, with $T_{\mathrm{s}}=1 / f_{\mathrm{s}}$ and $f_{\mathrm{s}}$ being the converter switching frequency. Considering the above time delay, the WT equivalent harmonic impedance of the positive-sequence harmonic components in (10) is rewritten as

$$
\underline{Z}_{\mathrm{WT}, \mathrm{p} k}^{(D)}=\frac{R_{\mathrm{f}}+j L_{\mathrm{f}}(h+1) \omega_{1}+\underline{D}_{h} \underline{H}_{i, h}\left(\underline{F}_{\mathrm{PI}, h}-j L_{\mathrm{f}} \omega_{1}\right)}{1-\underline{D}_{h} \underline{H}_{v, h}}
$$

where

$$
\begin{aligned}
& \underline{D}_{h}=\mathrm{e}^{-\mathrm{j} h \omega_{1} T_{\mathrm{d}}}=D_{R h}-\mathrm{j} D_{I h}, \quad D_{R h}=\cos \left(h \omega_{1} T_{\mathrm{d}}\right) \\
& \quad D_{I h}=\sin \left(h \omega_{1} T_{\mathrm{d}}\right) .
\end{aligned}
$$

Simplified expressions of the WT equivalent harmonic impedance (26) are difficult to obtain due to the addition of converter time delay. However, approximate expressions can be obtained in some conditions related to line current and grid voltage filtering.

In the case of unfiltered line currents (case B in Section 3.1.2), the following approximate positive-sequence harmonic components of the equivalent impedances can be determined from (26): (see (28))

If the grid voltage harmonics are unfiltered (i.e. $H_{v, h} \simeq 1$ ), (28) can be simplified as

$$
\begin{aligned}
& \underline{Z}_{\mathrm{WT}, k} \simeq L_{\mathrm{f}} \frac{\alpha_{\mathrm{c}}\left(D_{R h}-1\right)+h \omega_{1} D_{I h}}{2\left(1-D_{R h}\right)} \\
&+\mathrm{j} L_{\mathrm{f}}\left(\frac{h \omega_{1}}{2}+\frac{\alpha_{\mathrm{c}} D_{I h}}{2\left(1-D_{R h}\right)}\right) \\
&(h=k-1 ; \quad k=4,7),
\end{aligned}
$$

where according to Section 3.1.1, the WT equivalent harmonic impedance becomes $\underline{Z}_{\mathrm{WT}, k}^{(A)}=\infty(k=4,7 \ldots)$ when the time delay is equal to zero (i.e. $D_{R h}=1$ and $D_{I h}=0$ ).

If the grid voltage harmonics are filtered with a low bandwidth (approximately, $\alpha_{f \mathrm{v}, r}<5$ ), (28) can be simplified as

$$
\begin{aligned}
& \underline{Z}_{\mathrm{WT}, k} \simeq L_{\mathrm{f}}\left(\alpha_{\mathrm{c}}+\alpha_{\mathrm{f} v}\right) D_{R h}+\mathrm{j} L_{\mathrm{f}} h^{2} \omega_{1}^{2} \\
& \frac{h \omega_{1}+\left(\alpha_{\mathrm{f} v}-\alpha_{\mathrm{c}}\right) D_{I h}}{h^{2} \omega_{1}^{2}+2 \alpha_{\mathrm{f} v} h \omega_{1} D_{I h}} \quad(h=k-1 ; k=4,7),
\end{aligned}
$$

850

$$
\begin{aligned}
& \underline{Z}_{\mathrm{WT}, k} \simeq R_{\mathrm{WT}, k}+\mathrm{j} X_{\mathrm{WT} a, k} \quad(h=k-1 ; \quad k=4,7) \\
& R_{\mathrm{WT}, k}=L_{\mathrm{f}}\left(\frac{h \omega_{1}\left(\left(\alpha_{\mathrm{f} v}+\alpha_{\mathrm{c}}\right) h \omega_{1} D_{R h}+\alpha_{\mathrm{f} v}^{2} D_{I h}\right)+\alpha_{\mathrm{f} v}^{2} \alpha_{\mathrm{c}}\left(D_{R h}-1\right)}{\alpha_{\mathrm{f} v}^{2}\left(1-D_{R h}\right)^{2}+\left(h \omega_{1}+\alpha_{\mathrm{f} v} D_{I h}\right)^{2}}\right) \\
& X_{\mathrm{WT}, k}=L_{\mathrm{f}}\left(\frac{h^{3} \omega_{1}^{3}+h^{2} \omega_{1}^{2}\left(\alpha_{\mathrm{f} v}-\alpha_{\mathrm{c}}\right) D_{I h}+h \omega_{1} \alpha_{\mathrm{f} v}\left(\alpha_{\mathrm{f} v}\left(1-D_{R h}\right)-\alpha_{\mathrm{c}}\right)-\alpha_{\mathrm{f} v}^{2} \alpha_{\mathrm{c}} D_{I h}}{\alpha_{\mathrm{f} v}^{2}\left(1-D_{R h}\right)^{2}+\left(h \omega_{1}+\alpha_{\mathrm{f} v} D_{I h}\right)^{2}}\right) .
\end{aligned}
$$



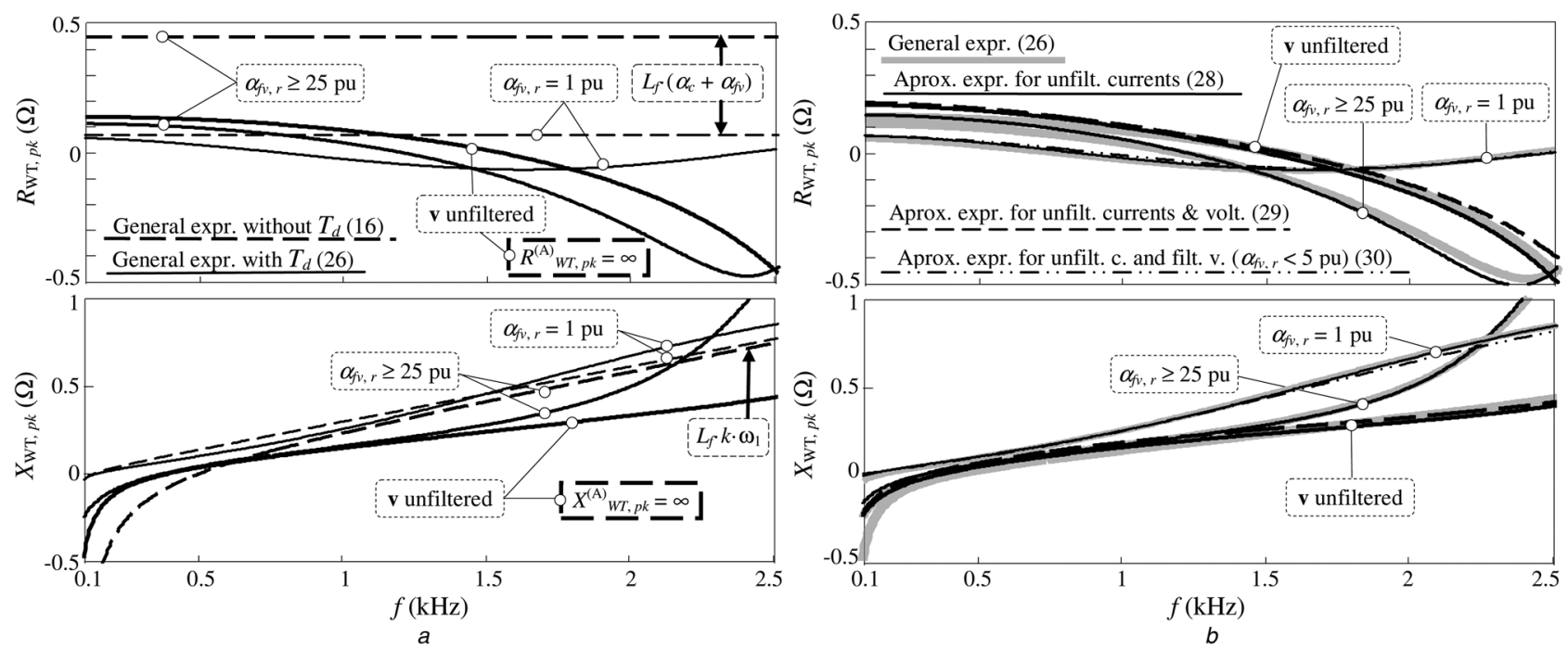

995

Fig. 5 Study of the WT positive-sequence equivalent resistance (up) and reactance (down) with unfiltered line currents (data in Table 1)

$a$ Comparison between equivalent impedances without and with converter time delay $\left(D(s)=1\right.$ and $D(s)=\mathrm{e}^{-s T_{\mathrm{d}}}$, respectively)

$b$ Comparison between general and approximate equivalent impedances with converter time delay $\left(D(s)=\mathrm{e}^{-s T_{\mathrm{d}}}\right.$ ' where according to Section 3.3.1, the WT equivalent harmonic impedance becomes (21) when the time delay is equal to zero (i.e. $D_{R h}=1$ and $\left.D_{I h}=0\right)$.

The WT equivalent harmonic impedances of the negative-sequence harmonic components $(k=2,5 \ldots)$ correspond to the conjugate expression of the positive-sequence WT equivalent harmonic impedances, with $h=-(k+1)$.

To study the impact of converter time delay on the WT equivalent harmonic impedance and analyse the accuracy of the previous expressions, Fig. 5 shows the positive-sequence WT equivalent resistance and reactance $\left(R_{\mathrm{WT}, k}\right.$ and $X_{\mathrm{WT}, k}$ with $\left.k=4,7 \ldots\right)$ for unfiltered line currents and three feedforward grid voltage cases (filtered grid voltage with $\alpha_{\mathrm{fv}, r}=1 \mathrm{pu}$ or $\alpha_{\mathrm{fv}, r}=25 \mathrm{pu}$ and unfiltered

In Fig. $5 a$, the general expressions of the equivalent impedances without and with converter time delay, (16) and (26), respectively, are compared with determine the influence of converter time delay. It can be noted that, if the grid voltage is filtered, converter time delay reduces the WT equivalent resistance, but does not change the $L_{\mathrm{f}} k \omega_{1}$ trend of the WT equivalent reactance significantly. This mainly reduces the damping effect of WT control at a frequency range where the WT equivalent resistance is smaller, but does not affect WPP resonance frequencies significantly. Moreover, it can be observed that converter time delay may lead to negative values of the WT equivalent resistance, worsening system stability [16]. If the grid voltage is unfiltered, the WT equivalent harmonic impedance is not infinite, as in the case of zero time delay (see Section 3.1.1), which affects the damping and frequency of WPP resonances. These conclusions are presented in Section 4.1. In Fig. 5b, the general expression of the equivalent impedance with converter time delay (26) is compared with the approximate expressions (28)-(30) to validate the accuracy of these approximations. Although the comparison of the WT equivalent harmonic impedances when the currents are filtered with $\alpha_{\mathrm{fi}, r}=15 \mathrm{pu}$ is not shown, it was verified that the approximate expressions do not exhibit the same accuracy, but can provide an acceptable prediction of the impedances because these impedances do not change significantly compared with the unfiltered case.

\section{Application to WPP resonances}

An $8 \times 5$ offshore WPP (data in Table 1) is studied to analyse the influence of WTs on WPP harmonic behaviour. The WPP consists of 40 type-4 WTs (i.e. full-scale VSC WTs), each with a rated capacity of $5 \mathrm{MW}$, arranged in five strings of $33 \mathrm{kV}$ grid voltage). submarine cables. These strings collect eight WTs (separated 1 $\mathrm{km}$ from each other) at the offshore substation, which is connected to shore by a $10 \mathrm{~km}$ submarine cable. According to Table 1, the short-circuit impedance of the grid is $\mathrm{SCR}=2500$ / $(8 \cdot 5 \cdot 5)=12.5 \mathrm{pu}$.

\subsection{Frequency response of the WPP}

The frequency response of the $8 \times 5$ offshore WPP is determined to analyse the influence of WTs on resonance frequencies. The frequency scan study is performed considering the ideal current source model in the literature (case A in Section 3.1), the general model of the WT equivalent harmonic impedance, (16), and the simplified models proposed in Section 3.3.3. The results of the WT positive-equivalent harmonic impedance considering zero time delay are illustrated for unfiltered and filtered $\left(\alpha_{\mathrm{f} i, r}=15 \mathrm{pu}\right)$ line current and several $\alpha_{\mathrm{fv}, r}$ values in Fig. 6 . These results show that the simplified models provide a good approximation of the general model. These simplified models allow easy analysis of the influence of WTs on WPP resonance frequencies in harmonic studies. It can be observed that WT control shifts WPP resonance frequencies and introduces a damping effect [9].

The damping effect of WT control is due to the resistance of the WT equivalent harmonic impedance, which depends on the filter inductance, $L_{\mathrm{f}}$, and current control and grid voltage low-pass filter bandwidths, $\alpha_{\mathrm{c}}$ and $\alpha_{\mathrm{fv}}$ (see Section 3.5). It is slightly greater in the unfiltered line current case than in the filtered line current case because of the additional term $L_{f} \cdot \alpha_{c}$ in the WT equivalent harmonic resistance. Moreover, it increases with increasing the grid voltage low-pass filter bandwidth because of the $L_{\mathrm{f}} \cdot \alpha_{\mathrm{fv}}$ term. Note that, in the filtered line current case, the approximation (25) can underestimate the damping effect at low frequencies if $\alpha_{\mathrm{fv}, r}$ is small (see $\alpha_{\mathrm{fi}, r}=15 \mathrm{pu}$ and $\alpha_{\mathrm{fv}, r}=1$ plot in Fig. 6). Hence, in this case it would be advisable to use (24). The accurate results provided by (24) for different values of the filter bandwidths were checked although they are not shown for the sake of clarity.

WT VSC control shifts the resonance frequencies as observed for $\alpha_{\mathrm{fv}, r}=1$. The influence of the filter capacitor $C_{\mathrm{f}}$ connected in parallel with the WTs is analysed in Fig. 7 to study this frequency response. Fig. 7 compares the impedances of the WT $\left(Z_{\mathrm{WT}, k}=R_{\mathrm{WT}, k}+j \cdot X_{\mathrm{WT}, k}\right)$, the filter capacitor $\left(Z_{C \mathrm{C}, k}=j \cdot X_{C \mathrm{C}, k}\right)$ and the WT-capacitor set $\left(Z_{\mathrm{WT} / / \mathrm{Cf}, k}=R_{\mathrm{WT} / \mathrm{Cf}, k}+j \cdot X_{\mathrm{WT} / \mathrm{Cf}, k}=Z_{\mathrm{WT}, k} / / Z_{\mathrm{Cf}, k}\right)$. It can be observed that there is a parallel resonance between the WT equivalent harmonic impedance and the filter capacitor reactances which, considering that $X_{\mathrm{WT}, k} \simeq L_{\mathrm{f}} \cdot k \cdot \omega_{1}$ and $X_{\mathrm{Cf}, k}=-1 /\left(C_{\mathrm{f}} \cdot k \cdot \omega_{1}\right)$,
1015 

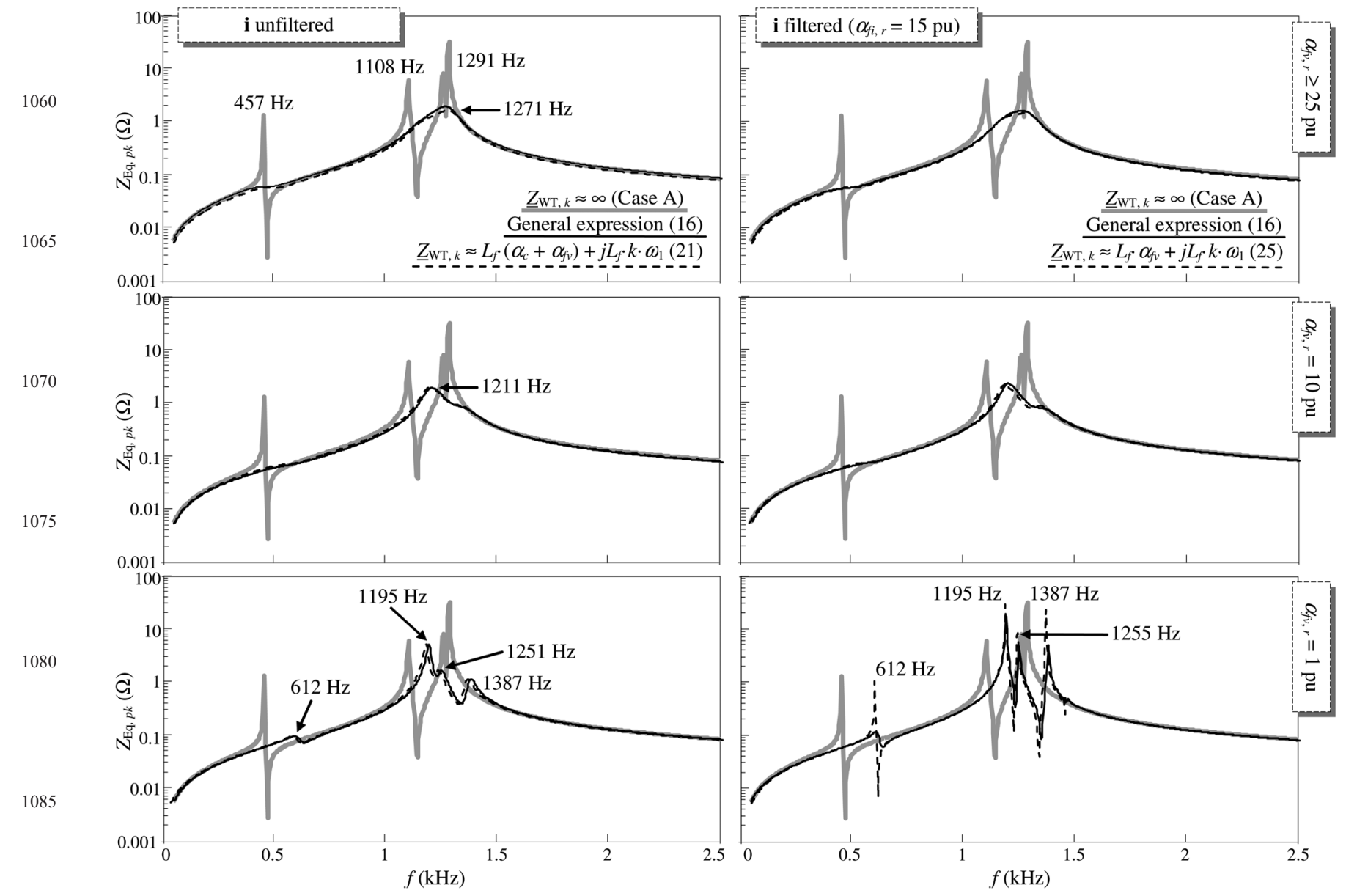

Fig. 6 Influence of WT VSC control on $8 \times 5$ offshore WPP resonance (data in Table 1 and D $(s)=1$ )

occurs approximately at the following frequency:

$$
f_{\text {res_Cf }} \simeq \frac{1}{2 \pi \sqrt{L_{\mathrm{f}} C_{\mathrm{f}}}} .
$$

According to Fig. 7, the WPP resonances below $f_{\text {res_Cf }}$ in Fig. 6 are shifted by the WTs because the frequency response of the WT-capacitor set is affected by the WT equivalent harmonic impedance response. On the other hand, the WPP resonances

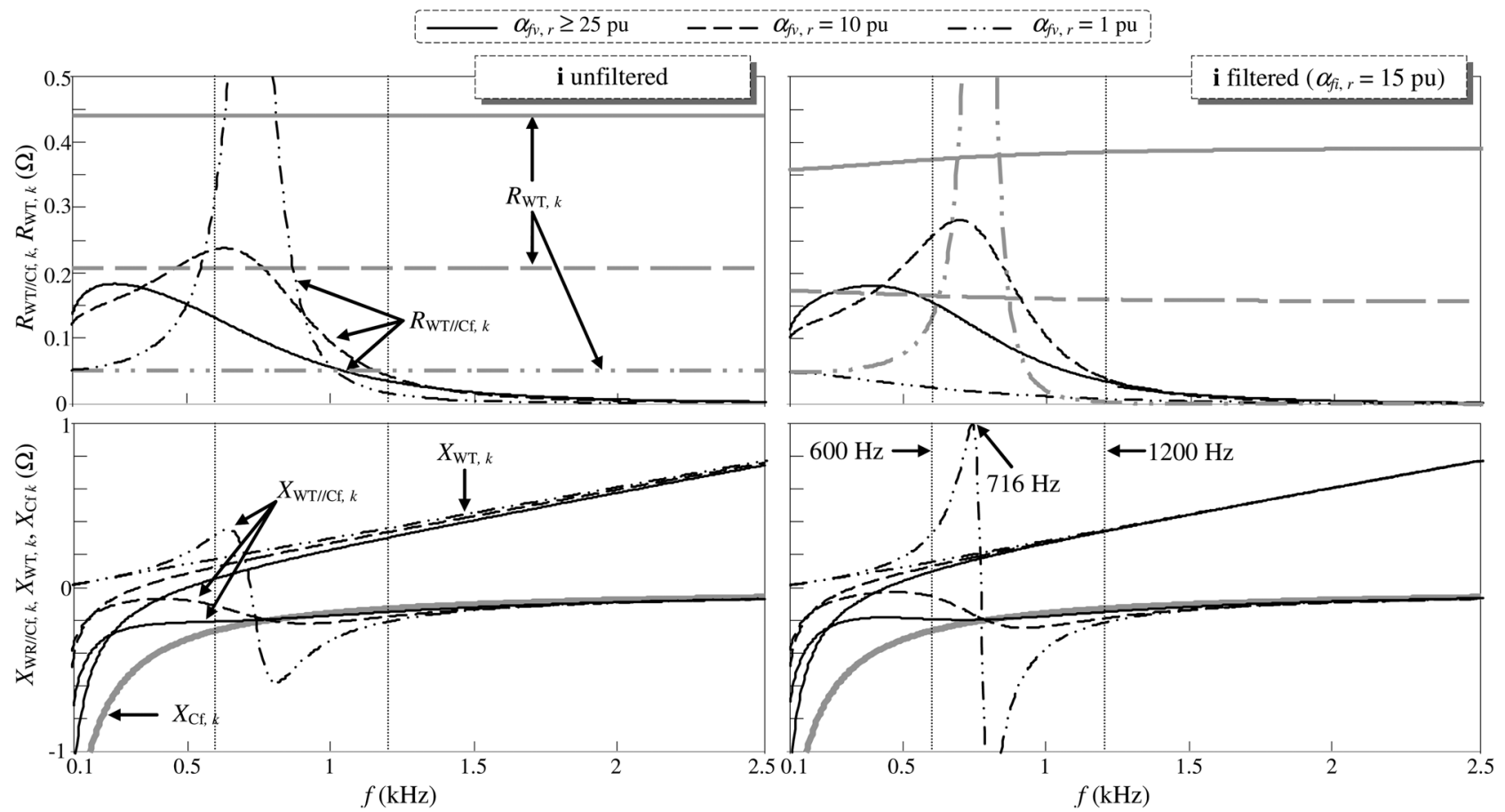

Fig. 7 Study of filter capacitor influence on WT equivalent impedance (data in Table 1 and $D(s)=1$ ) 

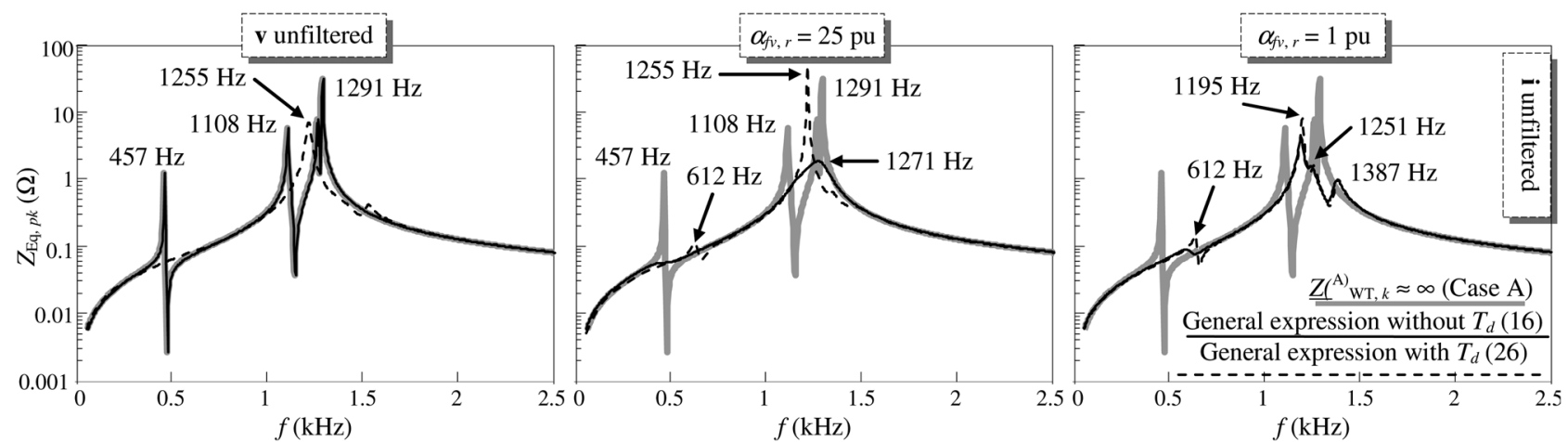

Fig. 8 Influence of WT VSC control with $D(s)=1$ or $D(s)=\mathrm{e}^{-s T_{\mathrm{d}}}$ on $8 \times 5$ offshore WPP resonance (data in Table 1)
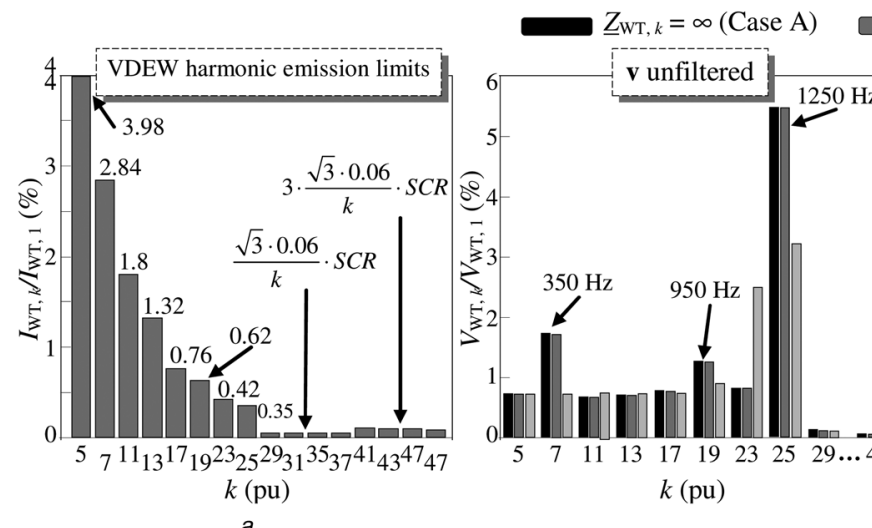

General expr. without $T_{d}(16)$

$\square$ General expr. with $T_{d}$ (26)

a

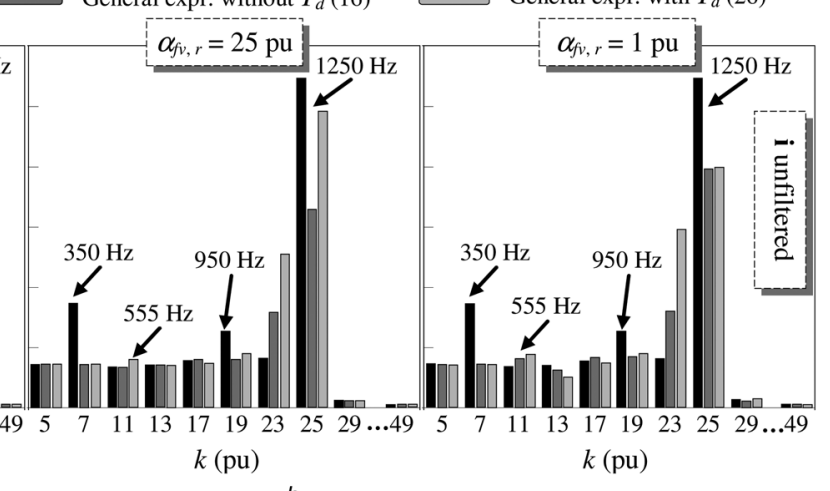

Fig. 9 Harmonic distortion at WT terminals (data in Table 1)

$a$ VDEW current emission limits

$b$ Voltage distortions

above $f_{\text {res_cf }}$ mainly depend on the filter capacitor although the parallel resonances in the example are still affected by the WTs. In these cases, the typical WT current source model could be adopted to analyse WPP harmonic response approximately if the WPP parallel resonances are far enough from $f_{\text {res_Cf }}$.

The comparison of the WT positive-equivalent harmonic impedance obtained from the WT ideal current source, zero time delay and non-zero time delay general models $\left(\underline{Z}_{W T, k}^{(A)}=\infty, \underline{Z}_{W T, k}^{(C)}\right.$ in (16) and $\underline{Z}_{\mathrm{WT}, k}^{(D)}$ in (26), respectively) is given for unfiltered line current and three feedforward grid voltage cases (filtered grid voltage with $\alpha_{\mathrm{fv}, r}=$ $1 \mathrm{pu}$ or $\alpha_{\mathrm{fv}, r}=25 \mathrm{pu}$ and unfiltered grid voltage) in Fig. 8. The results in Section 3.4.2 show that the non-zero time delay model affects the WPP resonances as follows: (i) it can reduce the damping effect compared with the zero time delay model depending on the frequency range and the feedforward filter bandwidth when the grid voltage is filtered, but does not modify WPP resonance frequencies significantly; (ii) it introduces a damping effect and modifies the WPP resonance frequencies when the grid voltage is unfiltered. Although the results with the approximate expressions of the non-zero time delay model, (29) and (30), are not plotted in Fig. 8 for the sake of clarity, it was verified that these expressions are highly accurate. It was also verified that the influence of non-zero time delay when the line current is filtered is similar to when the line current is unfiltered and that the approximate models for unfiltered line current also provide acceptable results.

All the subsection comments can be extended to the WT negative-equivalent harmonic impedance.

\subsection{Harmonic voltage distortion of the WPP}

The harmonic voltage distortions $H D_{\mathrm{WT} v, k}=V_{\mathrm{WT}, k} / V_{\mathrm{WT}, 1}$ at bus $N_{r 1}$ (i.e. at $\mathrm{WT}_{N r 1}$ terminals where resonances are studied) are numerically obtained from Matlab/Simulink simulations considering the harmonic current limits in the German Electricity Association (VDEW) Standard for generators connected to medium-voltage networks [4], which are set in the study as the WT emission magnitudes $I_{\mathrm{WT}, k}$ (or $H D_{\mathrm{WT} i, k}=I_{\mathrm{WT}, k} / I_{\mathrm{WT}, 1}$, where $I_{\mathrm{WT}, 1}=P_{\mathrm{WT}, \mathrm{N}} /$ $\left(\sqrt{3} \cdot U_{\mathrm{WT}, \mathrm{N}}\right)$ and $P_{\mathrm{WT}, \mathrm{N}}$ and $U_{\mathrm{WT}, \mathrm{N}}$ are in Table 1$)$. This standard provides specific magnitude values of generator (or WT) harmonic limits based on the grid short-circuit ratio SCR, which can be easily included in the simulation programme. The limits for a short-circuit ratio close to the 15-20 range are quite similar to the distortion limits in IEEE Standard 15471 (in particular, harmonics $<1.5 \mathrm{kHz}$ ) [4]. The harmonic voltages are easily obtained by multiplying the limit harmonic currents of the VDEW Standard by the WPP equivalent harmonic impedance at bus $N_{\mathrm{r}} 1$, i.e. $V_{\mathrm{WT}, k}=Z_{\mathrm{Eq}, k} \cdot I_{\mathrm{WT}, k}$, where the WT equivalent harmonic impedances are considered in $Z_{\mathrm{Eq}, k}$ according to the previous sections. Fig. $9 a$ plots the VDEW current emission limits used in the simulations and Fig. $9 b$ plots the voltage distortions considering WT unfiltered line currents and three feedforward grid voltage cases (unfiltered grid voltage and filtered grid voltage with $\alpha_{\mathrm{fv}, r}=25 \mathrm{pu}$ or $\alpha_{\mathrm{fv}, r}=1 \mathrm{pu}$ ). These voltage distortions are calculated considering the WT ideal current source model (i.e. $\underline{Z}_{\mathrm{Eq}, k}^{(A)}=\infty$ ) and the WT general model without and with time delay, $\underline{Z}_{\mathrm{Eq}, k}^{(C)}(16)$ and $\underline{Z}_{\mathrm{Eq}, k}^{(D)}(26)$, respectively. The harmonic frequencies with peak values of the voltage distortions are labelled to show that the voltage distortion pattern obtained is similar to the WPP harmonic response in Fig. 8. It can be observed that the frequencies of the maximum distortion values approximately correspond to the parallel resonance frequencies: (i) in the unfiltered grid voltage example, the peak values of voltage distortion at 350 and $950 \mathrm{~Hz}$ are related to the WPP equivalent impedance resonances at 475 and $1108 \mathrm{~Hz}$ of the ideal current source model 
and the peak values at $1250 \mathrm{~Hz}$ are related to the resonances in the $1255-1300 \mathrm{~Hz}$ range of all models; (ii) in the filtered grid voltage example with $\alpha_{\mathrm{fv}, r}=25 \mathrm{pu}$, the small peak value of voltage distortion at $555 \mathrm{~Hz}$ is related to the low resonance at $612 \mathrm{~Hz}$ of the non-zero time delay model and the other peak values are the same as in the unfiltered grid voltage example; (iii) in the filtered grid voltage example with $\alpha_{\mathrm{fv}, r}=1 \mathrm{pu}$, the results are the same as with $\alpha_{\mathrm{fv}, r}=25 \mathrm{pu}$ but the peak value of voltage distortion at $555 \mathrm{~Hz}$ is greater because the resonance at $612 \mathrm{~Hz}$ of the non-zero time delay model is higher. The results show that harmonic voltage

1330 distortions can be higher and more dangerous when the parallel resonance matches exactly some frequencies of the WT harmonic emissions than when it is close to them. According to Section 3, the results of the general model without time delay (16) are similar to the results of the ideal current source model (case A in Section 3.1.1) when the grid voltage is unfiltered and they can also be approximated with (21) when the grid voltage is filtered. The results of the general model with time delay (26) can be approximated with (29) or (30) when the grid voltage is unfiltered or when it is filtered with a low bandwidth, respectively. It must be noted that the study is just an example to illustrate how the

1340 present work could be used in actual WPP applications to predict harmonic distortions at the WT connection point. Further research would be required to accurately evaluate the impact of resonance on WP power quality.

\section{Conclusion}

The influence of WT VSC control on offshore WPP harmonic response is studied. Analytical expressions of the WT equivalent

1350 harmonic impedance are presented considering the effect of current and feed-forward voltage filters. The influence of converter time delay in these expressions is also analysed. The obtained expressions allow the WT converter effect on WPP frequency scanning to be considered. Simplified expressions for the impedance which can be easily applied in frequency scanning are also proposed. It is observed that WT modelling as an ideal current source can lead to inaccurate results because WT VSC control shifts WPP resonance frequencies and introduces a damping effect. Frequency shifting must be considered for the whole frequency range. On the other hand, for frequencies above

1360 the resonance frequency between the WT filter inductance and filter capacitor, this shifting is mainly due to the filter capacitor. Therefore, the WT current source model could be used to estimate the range of parallel resonances if they are far from the WT filter resonance. The damping effect is also observed for the whole frequency range and is directly related to current control and grid voltage low-pass filter bandwidths. In general, converter time delay reduces the damping effect when the feedforward voltages are filtered, but does not affect resonance frequencies significantly. In the case of unfiltered feedforward voltages, converter time delay increases the damping effect, which may modify resonance

1370 frequencies. PSCAD and Matlab/Simulink simulations are used to validate the analytical study. It must be noted that other WT VSC control structures lead to different WT Norton equivalent impedances, and therefore their influence on WPP resonances will be different. This influence could, however, be analysed following the procedure described here.

\section{Acknowledgments}

1380 L. Sainz's work was carried out with the financial support of the 'Ministerio de Economía y Competitividad' (grant ENE2013-46205C5-3-R), which the authors gratefully acknowledge. M. CheahMane's work received funding from the People Programme (Marie Curie Actions) of the European Union Seventh Framework Programme FP7/2007-2013/ under REA grant agreement no. 317221, project title MEDOW.

\section{References}

1 Kocewiak, L.H.: 'Harmonics in large offshore wind farms'. Thesis for the $\mathrm{PhD}$ Degree in Electrical Engineering, Department of Energy Technology, Aalborg University, Denmark, 2012, http://vbn.aau.dk/files/62660098/lukasz_kocewiak.pdf

2 Papathanassiou, S.A., Papadopoulos, M.P.: 'Harmonic analysis in a power system with wind generation', IEEE Trans. Power Deliv., 2006, 21, (4), pp. 2006-2016

3 Tentzerakis, S.T., Papathanassiou, S.A.: 'An investigation of the harmonic emissions of wind turbines', IEEE Trans. Energy Conver., 2007, 22, (1), pp. $150-158$

4 Rockhill, A.A., Liserre, M., Teodorescu, R., et al.: 'Grid-filter design for a multimegawatt medium-voltage source inverter', IEEE Trans. Ind. Electron., 2013, 58, (4), pp. 1205-1216

5 IEEE Standard for interconnecting distributed resources with electric power systems. IEEE Standard 15471, 2005

6 IEEE PES Wind Plant Collector System Design Working Group.: 'Harmonics and resonance issues in wind power plants'. Proc. IEEE Power and Energy Society General Meeting, July 2011, pp. 1-8

7 Yang, K.: 'On harmonic emissions, propagation and aggregation a wind power plants'. Doctoral thesis, Department of Engineering Sciences and Mathematics, Lulea University of Technology, Sweden, http://pure.ltu.se/portal/en/

8 Axelsson, U., Holm, U., Bollen, M., et al.: 'Propagation of harmonic emission from the turbines through the collection grid to the public grid'. Proc. 22nd Int. Conf. Exhibition on Electricity Distribution (CIRED 2013), 2013, pp. 1-4

9 Buchhagen, C., Rauscher, C., Menze, A., et al.: 'BorWin1 - first experiences with harmonic interactions in converter dominated grids'. Int. ETG Congress, November 2015, pp. 1-7

10 Zheng, R., Bollen, M., Zhong, J.: 'Harmonic resonances due to a grid-connected wind farm'. Proc. 14th Int. Conf. Harmonics and Quality of Power (ICHQP 2010), September 2010, pp. 1-7

$11 \mathrm{Li}$, J., Samaan, N., Williams, S.: 'Modeling of large wind farm systems for dynamic and harmonics analysis'. Proc. IEEE/PES Transmission and Distribution Conf. and Exposition, April 2008

12 Ghassemi, F., Koo, K-L.: 'Equivalent network for wind farm harmonic assessments', IEEE Trans. Power Deliv., 2010, 25, (3), pp. 1808-1815

13 Zhang, S., Jiang, S., Lu, X., et al.: 'Resonance issues and damping techniques for grid-connected inverters with long transmission cable', IEEE Trans. Power Electron., 2014, 29, (1), pp. 110-120

14 Harnefors, L., Yepes, A.G., Vidal, A., et al.: 'Passivity-based controller design of grid-connected VSCs for prevention of electrical resonance instability', IEEE Trans. Indust. Electron., 2015, 62, (2), pp. 702-710

15 Chen, X., Sun, J.: 'A study of renewable energy systems harmonic resonance based on a DG test-bed'. Proc. 26th IEEE Applied Power Electronics Conf. and Exposition (APEC 2011), March 2011, pp. 995-1002

16 Harnefors, L., Bongiorno, M., Lundberg, S.: 'Input-admittance calculation and shaping for controlled voltage-source converters', IEEE Trans. Indust. Electron., 2007, 54, (6), pp. 3323-3334

17 Cespedes, M., Sun, J.: 'Modeling and mitigation of harmonic resonance between wind turbines and the grid'. Proc. IEEE Energy Conversion Congress and Exposition, September 2011, pp. 2109-2116

18 Cespedes, M., Sun, J.: 'Impedance modeling and analysis of grid-connected voltage-source converters', IEEE Trans. Power Electron., 2014, 29, (3), pp. 1254-1261

19 Freijedo, F.D., Chaudhary, S.K., Teodorescu, R., et al.: 'Harmonic resonances in wind power plants: modeling, analysis and active mitigation methods'. 2015 IEEE Eindhoven PowerTech, June 2015, pp. 1-6

20 Bakhshizadeh, M.K., Hjerrild, J., Kocewiak, L., et al.: 'Harmonic modelling, propagation and mitigation for large wind power plants connected via long HVAC cables: review and outlook of current research'. IEEE Int. Energy Conf. (ENERGYCON 2016), April 2016, pp. 1-5

21 Wang, X., Blaabjerg, F., Wu, W.: 'Modeling and analysis of harmonic stability in an AC power-electronics-based power system', IEEE Trans. Power Electron., 2014, 29, (12), pp. 6421-6432

22 Bayo-Salas, A., Beertenyz, J., Rimez, J., et al.: 'Impedance-based stability assessment of parallel VSC HVDC grid connections'. 11th IET Int. Conf. on AC and DC power transmission, February 2015 , pp. 1-9

23 Cespedes, M., Sun, J.: 'Three-phase impedance measurement for system stability analysis'. IEEE 14th Workshop on Control and Modeling for Power Electronics (COMPEL), June 2013, pp. 1-6

\section{Appendices}

\subsection{Complex space phasor representation of harmonics in synchronous (or $d-q$ ) reference frame}

If zero-sequence components are not considered, three-phase quantities $\left(x_{a}(t), x_{b}(t), x_{c}(t)\right)$ can be expressed as $d-q$ complex space phasors as follows

$$
\boldsymbol{x}=x_{d}+\mathrm{j} x_{q}=\frac{2}{3}\left(x_{a}+\mathrm{e}^{\mathrm{j} 2 \pi / 3} x_{b}+\mathrm{e}^{\mathrm{j} 4 \pi / 3} x_{c}\right) \mathrm{e}^{-\mathrm{j} \theta_{1}},
$$

where $\mathrm{d} \theta_{1} / \mathrm{d} t=\omega_{1}$. 
The results of transformation (32) depend on the unbalanced or balanced conditions of the three-phase quantities.

8.1.1 Unbalanced conditions: Considering (32), three-phase non-sinusoidal quantities in unbalanced conditions

$$
\begin{array}{r}
x_{a}(t)=\operatorname{Re}\left\{\underline{X}_{a 1} \cdot \mathrm{e}^{\mathrm{j} \omega_{1} t}\right\}+\sum_{k>1} \operatorname{Re}\left\{\underline{X}_{a k} \cdot \mathrm{e}^{\mathrm{j} k \omega_{1} t}\right\}, \\
x_{b}(t)=\operatorname{Re}\left\{\underline{X}_{b 1} \cdot \mathrm{e}^{\mathrm{j} \omega_{1} t}\right\}+\sum_{k>1} \operatorname{Re}\left\{\underline{X}_{b k} \cdot \mathrm{e}^{\mathrm{j} k \omega_{1} t}\right\}, \\
x_{c}(t)=\operatorname{Re}\left\{\underline{X}_{c 1} \cdot \mathrm{e}^{\mathrm{j} \omega_{1} t}\right\}+\sum_{k>1} \operatorname{Re}\left\{\underline{X}_{c k} \cdot \mathrm{e}^{\mathrm{j} k \omega_{1} t}\right\}
\end{array}
$$

are expressed as $d-q$ complex space phasors as follows

$$
\begin{aligned}
\boldsymbol{x}= & \underline{X}_{\mathrm{p} 1}+\underline{X}_{\mathrm{n} 1}^{*} \mathrm{e}^{-\mathrm{j} 2 \cdot \omega_{1} \cdot t} \\
& +\sum_{k>1}\left(\underline{X}_{\mathrm{p} k} \mathrm{e}^{\mathrm{j}(k-1) \cdot \omega_{1} \cdot t}+\underline{X}_{\mathrm{n} k}^{*} \mathrm{e}^{-\mathrm{j}(k+1) \cdot \omega_{1} \cdot t}\right),
\end{aligned}
$$

where the complex vectors $X_{\mathrm{p} 1}=X_{\mathrm{p} 1} \angle \phi_{\mathrm{p} 1}$ and $X_{\mathrm{n} 1}=X_{\mathrm{n} 1} \angle \phi_{\mathrm{n} 1}$ are the positive- and negative-sequence fundamental phasors and the complex vectors $X_{\mathrm{p} k}=X_{\mathrm{p} k} \angle \phi_{\mathrm{p} k}$ and $\underline{X}_{\mathrm{n} k}=X_{\mathrm{n} k} \angle \phi_{\mathrm{n} k}$ (with $k>1$ ) are the $k$ th positive- and negative-sequence harmonic phasors of the unbalanced three-phase non-sinusoidal quantities (superscript * indicates complex conjugate).
8.1.2 Balanced conditions: Three-phase non-sinusoidal quantities in balanced conditions

$$
\begin{aligned}
& x_{a}(t)=\operatorname{Re}\left\{\underline{X}_{1} \cdot \mathrm{e}^{\mathrm{j} \omega_{1} t}\right\}+\sum_{k>1} \operatorname{Re}\left\{\underline{X}_{k} \cdot \mathrm{e}^{\mathrm{j} k \omega_{1} t}\right\} \\
& x_{b}(t)=x_{a}\left(t+2 T_{1} / 3\right) \quad x_{c}(t)=x_{a}\left(t+T_{1} / 3\right) \quad\left(T_{1}=1 / f_{1}\right),
\end{aligned}
$$

verify that the complex vector $X_{1}=X_{1} \angle \phi_{1}$ is the fundamental positive-sequence phasor of the balanced three-phase quantities (i.e. under balanced conditions, $X_{\mathrm{p} 1}=X_{1}$ and $X_{\mathrm{n} 1}=0$ in (34)) and the complex vectors $X_{k}=X_{k} \angle \phi_{k}$ (with $k=4, \overline{7} \ldots$ and $k=2,5 \ldots$ ) are the $k$ th positive- and negative-sequence harmonic phasors of the balanced three-phase quantities (i.e. under balanced conditions, $X_{\mathrm{p} k}=X_{k}$ and $X_{\mathrm{n} k}=0$ for $k=4,7 \ldots$ and $X_{\mathrm{p} k}=0$ and $X_{\mathrm{n} k}=X_{k}$ for $k=$ $2,5 \ldots$ in (34)). Thus, considering (32), three-phase non-sinusoidal quantities under balanced conditions are expressed as $d-q$ complex space phasors as follows

$$
\boldsymbol{x}=\underline{X}_{1}+\sum_{k=4,7 \ldots} \underline{X}_{k} \mathrm{e}^{\mathrm{j}(k-1) \cdot \omega_{1} \cdot t}+\sum_{k=2,5 \ldots} \underline{X}_{k}^{*} \mathrm{e}^{-\mathrm{j}(k+1) \cdot \omega_{1} \cdot t} .
$$

Note that, under balanced conditions, harmonics with orders of multiples of 3 (i.e. $k=3,6 \ldots$ ) correspond to the zero-sequence phasors, which are zero in $d-q$ reference frame and are usually neglected in WPP applications. Even harmonics are also negligible in power systems because of voltage and current half-wave symmetry. 


\section{${ }^{1585}$ EPA20160241}

Author Queries

Luis Sainz, Lluis Monjo, Joaquin Pedra, Marc Cheah-Mane, Jun Liang, Oriol Gomis-Bellmunt

1590 Q1 Please check the expansion of forename for all authors.

Q2 Please expand the acronym VSC

Q3 Please provide URL for Ref. [5]. 